\title{
Dynamics of a Predator-Prey Model with Fear Effect and Time Delay
}

\author{
Junli Liu $\mathbb{D}^{1},{ }^{1}$ Pan Lv, ${ }^{1}$ Bairu Liu, ${ }^{1}$ and Tailei Zhang ${ }^{2}$ \\ ${ }^{1}$ School of Science, Xi'an Polytechnic University, Xi'an 710048, China \\ ${ }^{2}$ School of Science, Chang'an University, Xi'an 710064, China \\ Correspondence should be addressed to Junli Liu; jlliu2008@126.com
}

Received 19 June 2020; Revised 26 March 2021; Accepted 13 April 2021; Published 28 April 2021

Academic Editor: Eulalia Martinez

Copyright $@ 2021$ Junli Liu et al. This is an open access article distributed under the Creative Commons Attribution License, which permits unrestricted use, distribution, and reproduction in any medium, provided the original work is properly cited.

In this paper, we propose a time-delayed predator-prey model with Holling-type II functional response, which incorporates the gestation period and the cost of fear into prey reproduction. The dynamical behavior of this system is both analytically and numerically investigated from the viewpoint of stability, permanence, and bifurcation. We found that there are stability switches, and Hopf bifurcations occur when the delay $\tau$ passes through a sequence of critical values. The explicit formulae which determine the direction, stability, and other properties of the bifurcating periodic solutions are given by using the normal form theory and center manifold theorem. We perform extensive numerical simulations to explore the impact of some important parameters on the dynamics of the system. Numerical simulations show that high levels of fear have a stabilizing effect while relatively low levels of fear have a destabilizing effect on the predator-prey interactions which lead to limit-cycle oscillations. We also found that the model with or without a delay-dependent factor can have a significantly different dynamics. Thus, ignoring the delay or not including the delay-dependent factor might result in inaccurate modelling predictions.

\section{Introduction}

Predator-prey interaction is a central topic in ecology and evolutionary biology which has been extensively studied from different aspects by many researchers over the last few decades. Mathematical models have played a vital role in understanding these complex scenarios. Most of the existing predator-prey models are based upon classical Lotka-Volterra formalism, and it is usually assumed that the predators can impact the prey population only through direct killing as direct predation is relatively easy to observe in nature. However, the presence of predator may significantly change the behaviour and physiology of prey to such an extent that it could affect the prey population more effective than direct predation [1-3].

In the presence of predators, many prey species change their behaviour due to predation risk and show a variety of antipredator responses, which incorporates foraging activity [4], habitat alterations, vigilance [5] and some physiological changes, etc. $[3,6]$. Many researchers observed that due to fear of predation risk, the reproduction of the scared prey decreases. For example, in the Greater Yellowstone Ecosystem, wolves influence the reproductive physiology of Elks [7]. Snowshoe hares shift to relatively safe but less profitable microhabitats [8]. Birds react to the sound of predator species with antipredator defenses, and they flee from their nests at the first sight of danger [1]. Other pieces of evidence suggest that fear can also affect populations like mule deer [9] and dugongs [10].

In 2011, Zanette et al. conducted a manipulation on song sparrows during a whole breeding season and showed that there is a $40 \%$ reduction in the offspring production of the song sparrows due to the fear from predator [11]. Very few researchers have investigated the impact of fear effect in a predator-prey model with the help of mathematical modelling. Wang et al. first proposed a predator-prey model by incorporating the cost of fear into prey production. Their analysis showed that fear effect can stabilize the oscillation of the system [12]. Wang and Zou formulated a predator-prey model with the cost of fear and adaptive avoidance of 
predators. The prey population is divided into juvenile and mature stages; this model is represented by a system of delay differential equations with maturation delay [13]. They theoretically analyzed the model dynamics for constant defense level and adaptive defense level. Numerical simulations showed that both strong adaptation of adult prey and the large cost of fear have destabilizing effect, but large population of predators has a stabilizing effect on the system. Sasmal and Takeuchi studied a predator-prey system with fear effect, group defense, and Holling-type IV functional response; they observed that the model can exhibit multistability [14]. Panday et al. studied the impact of fear in a tritrophic food chain model with Holling-type II functional response and observed that fear can stabilize the system from chaos to stable focus through the period-halving phenomenon, and chaotic dynamics can be controlled by the fear factors [15]. Sarkar and Khajanchi [16] proposed and analyzed a prey-predator system introducing the cost of fear into prey reproduction with Holling-type II functional response. Their mathematical analysis suggests that strong antipredator responses can stabilize the prey-predator interactions by ignoring the existence of periodic behaviors.

Inspired by the aforementioned works, the aim of the current work is to develop a predator-prey model, which incorporates the fear effects of predators on prey as well as the time delay. Further, we investigate the impacts of important parameters on the dynamics of system. The rest of the paper is organized as follows. In the next section, we will give the underlying assumptions and formulate the model. In the following section, we present some results on the stability of equilibria and existence of Hopf bifurcation. In Section 4, we investigate the properties of the bifurcating periodic solutions by using the normal form theory and center manifold theorem. We show the permanence of the system in Section 5. In Section 6, we provide some numerical simulation results which reveal some potential roles that the fear effect and the time delay may play in predator-prey interactions. A brief discussion completes the paper.

\section{Model Formulation}

Let $x(t)$ and $y(t)$ be the densities of prey and predator populations at time $t$, respectively. We assume that the prey population follows a logistic growth without any predator population, which can be split into three different parts, namely, the birth rate, the natural death rate, and the density dependent death due to intraspecific competition among preys. Let $r$ be the birth rate of prey, $d_{1}$ be the natural death rate of prey, and $d_{2}$ be the death rate due to intraprey competition. Since the fear effect will reduce the production, we will multiply the production term of prey $r x$ by a factor $f(k, y)=(1 /(1+k y))$ which accounts for the cost of antipredator defense due to predation fear. Also, it is reasonable to assume that the production of predators after predation is not instantaneous; for the sake of simplicity, we suppose that the time lag required for gestation of predator is $\tau$. Therefore, our predator-prey model with fear effect is given by

$$
\left\{\begin{array}{l}
\frac{\mathrm{d} x}{\mathrm{~d} t}=\frac{r x}{1+k y}-\mathrm{d}_{1} x-\mathrm{d}_{2} x^{2}-\frac{m x y}{1+a x} \\
\frac{\mathrm{d} y}{\mathrm{~d} t}=\frac{m c e^{-d \tau} x(t-\tau) y(t-\tau)}{1+a x(t-\tau)}-\mathrm{d} y .
\end{array}\right.
$$

Here, the parameter $k$ refers to the level of fear, which is due to the antipredator response of prey. In this study, we adopt the widely used Holling-type II functional response of predators [17], where $(m / a)$ is the predation rate and $(1 / a)$ is the half-saturation constant. $c$ is the conversion efficiency from prey biomass to predator biomass and $d$ is the death rate of predator. Since predator goes through a gestation stage, and it endures the death rate, then a factor $e^{-d \tau}$ appears in the first term of the second equation of system (1). The parameters $r, d_{1}, d_{2}, m, a, c$, and $d$ are all positive constants, and $k$ and $\tau$ are nonnegative constants.

The initial conditions take the form of

$$
(x(\theta), y(\theta))=\left(\phi_{1}(\theta), \phi_{2}(\theta)\right) \in C\left([-\tau, 0], R_{+}^{2}\right),
$$

where $C\left([-\tau, 0], R_{+}^{2}\right)$ denotes the space of continuous functions mapping the interval $[-\tau, 0]$ into $R_{+}^{2}$ and denotes the norm of an element $\phi=\left(\phi_{1}, \phi_{2}\right)$ in $C\left([-\tau, 0], R_{+}^{2}\right)$ by $\|\phi\|=\sup _{-\tau \leq \theta \leq 0}\left\{\left|\phi_{1}(\theta)\right|,\left|\phi_{2}(\theta)\right|\right\}$.

It is straightforward to show (see [18]) the following.

Lemma 1. The solution of system (1) with an initial condition (2) is unique and nonnegative for all $t \geq 0$.

Note that from the first equation in (1), we obtain $(d x / d t) \leq\left(r-d_{1}\right) x$; by a comparison argument, we see that if $r<d_{1}$, then $\lim _{t \rightarrow \infty} x(t)=0$, and applying the theory of asymptotically autonomous systems to the second equation in (1), we also have $\lim _{t \rightarrow \infty} y(t)=0$. That is, when $r<d_{1}$, both prey and predator species go to extinction. So, throughout this paper, we assume that $r>d_{1}$.

Lemma 2. There exists an $M>0$ such that for any solution of system (1) with an initial condition (2), there must be a $T>0$ such that $x(t) \leq M$ and $y(t) \leq M$ for $t \geq T$.

Proof. We first consider the system

$$
\frac{\mathrm{d} z}{\mathrm{~d} t}=\left(r-d_{1}\right) z-\mathrm{d}_{2} z^{2}
$$

whose solution satisfies

$$
\limsup _{t \rightarrow \infty} z(t) \leq \frac{r-\mathrm{d}_{1}}{\mathrm{~d}_{2}} .
$$

Since $(\mathrm{d} x / \mathrm{d} t) \leq\left(r-\mathrm{d}_{1}\right) x-\mathrm{d}_{2} x^{2}$, then by the comparison principle, we have

$$
\limsup _{t \rightarrow \infty} x(t) \leq \frac{r-d_{1}}{d_{2}} .
$$

Hence, for any $\varepsilon>0$ small enough, there exists a $t^{*}>0$ sufficiently large such that 


$$
x(t) \leq \frac{r-\mathrm{d}_{1}}{\mathrm{~d}_{2}}+\varepsilon \text { for } t>t^{*} .
$$

For $L(t)=c e^{-d \tau} x(t-\tau)+y(t)$ and $t>t^{*}+\tau$, we have

$$
\begin{aligned}
\frac{\mathrm{d} L(t)}{\mathrm{d} t}= & c e^{-d \tau}\left[\frac{r x(t-\tau)}{1+k y(t-\tau)}-\mathrm{d}_{1} x(t-\tau)-\mathrm{d}_{2} x^{2}(t-\tau)\right] \\
& -\mathrm{d} y(t), \\
\leq & c e^{-d \tau}\left(r-\mathrm{d}_{1}\right) x(t-\tau)-\mathrm{d} y(t) \\
= & c e^{-d \tau}\left(r-\mathrm{d}_{1}+\mathrm{d}\right) x(t-\tau)-\mathrm{d} L(t) \\
\leq & c e^{-d \tau}\left(r-\mathrm{d}_{1}+\mathrm{d}\right)\left(\frac{r-\mathrm{d}_{1}}{\mathrm{~d}_{2}}+\varepsilon\right)-\mathrm{d} L(t) .
\end{aligned}
$$

It follows that

$\limsup _{t \rightarrow \infty} L(t) \leq \frac{c e^{-\mathrm{d} \tau}\left(r-\mathrm{d}_{1}+\mathrm{d}\right)\left(\left(\left(r-\mathrm{d}_{1}\right) / \mathrm{d}_{2}\right)+\varepsilon\right)}{\mathrm{d}}$.

This completes the proof.

Lemma 3 (see [19], Lemma 2.1). Consider the following equation:

$$
\dot{x}(t)=a x(t-\tau)-b x(t)
$$

wherea, $b, \tau>0 ; x(t)>0$ for $-\tau \leq t \leq 0$. We have

(i) if $a<b$, then $\lim _{t \rightarrow+\infty} x(t)=0$

(ii) if $a>b$, then $\lim _{t \longrightarrow+\infty} x(t)=+\infty$

It is easy to check that (1) has a trivial extinction equilibrium $E_{0}=(0,0)$. This system also has a semitrivial equilibrium $E_{1}=\left(\left(\left(r-d_{1}\right) / d_{2}\right), 0\right)$ under the condition $r>d_{1}$. To find the positive (coexistence) equilibrium $E^{*}=\left(x^{*}, y^{*}\right)$, letting the right-hand side be zero, we then get $x^{*}=\left(d /\left(m c e^{-d \tau}-d a\right)\right)$, which exists if $m c e^{-d \tau}>d a$, and $y^{*}$ is a positive root of the equation

$$
a_{0} y^{2}+a_{1} y+a_{2}=0
$$

where $a_{0}=m k, a_{1}=m+k\left(d_{1}+d_{2} x^{*}\right)\left(1+a x^{*}\right), a_{2}=\left(d_{1}+\right.$ $\left.d_{2} x^{*}-r\right)\left(1+a x^{*}\right)$. Clearly, (10) has one positive root $y^{*}$ if and only if $d_{1}+d_{2} x^{*}-r<0$; this inequality is equivalent to

$$
m c e^{-d \tau}>\mathrm{d} a+\frac{\mathrm{d} d_{2}}{r-\mathrm{d}_{1}}
$$

Then, we conclude that (1) has a unique positive equilibrium $E^{*}=\left(x^{*}, y^{*}\right)$ if (11) holds, and the expression for $y^{*}$ is as follows:

$$
y^{*}=\frac{-a_{1}+\sqrt{a_{1}^{2}-4 a_{0} a_{2}}}{2 a_{0}} .
$$

\section{Stability Analysis and Hopf Bifurcation}

Theorem 1. The trivial extinction equilibrium $E_{0}=(0,0)$ is always an unstable saddle point. The semitrivial equilibrium $E_{1}=\left(\left(\left(r-d_{1}\right) / d_{2}\right), 0\right)$ is globally asymptotically stable in $\widetilde{C}=$ $\left\{\phi_{1}(\theta), \phi_{2}(\theta) \in C\left([-\tau, 0], R_{+}^{2}\right) \mid \phi_{1}(0)>0\right\}$ if $(11)$ is reversed and is unstable if (11) holds; meanwhile, $E^{*}$ exists.

Proof. For equilibrium $E_{0}=(0,0)$, the characteristic equation is

$$
\left(\lambda+d_{1}-r\right)(\lambda+\mathrm{d})=0 .
$$

Obviously, (13) has one positive root $r-d_{1}$ and one negative root $-m$. Hence, $E_{0}$ is a saddle point.

The characteristic equation at $E_{1}=\left(\left(\left(r-\mathrm{d}_{1}\right) / \mathrm{d}_{2}\right), 0\right)$ is as follows:

$$
\left(\lambda+r-\mathrm{d}_{1}\right)\left(\lambda+d-\frac{m c e^{-\mathrm{d} \tau}\left(r-\mathrm{d}_{1}\right)}{\mathrm{d}_{2}+a\left(r-\mathrm{d}_{1}\right)} e^{-\lambda \tau}\right)=0 .
$$

Let $f(\lambda)=\lambda+d-\left(\left(m c e^{-d \tau}\left(r-\mathrm{d}_{1}\right)\right) /\left(\mathrm{d}_{2}+a\left(r-\mathrm{d}_{1}\right)\right)\right)$ $e^{-\lambda \tau}$; then it is clear that (14) has one root $d_{1}-r<0$ and the other roots are determined by the equation $f(\lambda)=0$.

If (11) is reversed, then $d>\left(\left(m c e^{-d \tau}\left(r-d_{1}\right)\right) /\right.$ $\left.\left(d_{2}+a\left(r-d_{1}\right)\right)\right)$. Let $\lambda=u+i v$ with $u, v \in \mathbb{R}$ be a root of $f(\lambda)=0$. Then, we have

$$
(u+d)^{2}+v^{2}=\left(\frac{m c e^{-\mathrm{d} \tau}\left(r-\mathrm{d}_{1}\right) e^{-u \tau}}{d_{2}+a\left(r-\mathrm{d}_{1}\right)}\right)^{2} .
$$

If $u \geq 0$, then

$$
(u+d)^{2}+v^{2}>\left(\frac{m c e^{-d \tau}\left(r-\mathrm{d}_{1}\right)}{d_{2}+a\left(r-\mathrm{d}_{1}\right)}\right)^{2} \geq\left(\frac{m c e^{-d \tau}\left(r-\mathrm{d}_{1}\right) e^{-u \tau}}{d_{2}+a\left(r-\mathrm{d}_{1}\right)}\right)^{2} .
$$

A Contradiction. This shows that all roots of $f(\lambda)=0$ must have negative real parts. Therefore, $E_{1}$ is locally asymptotically stable.

Next, we will show that $E_{1}$ is globally attractive if (11) is reversed. Since $m c e^{-d \tau}<\mathrm{d} a+\left(\mathrm{dd}_{2} /\left(r-d_{1}\right)\right)$, we can choose small enough $\varepsilon>0$ such that

$$
\frac{m c e^{-d \tau}\left(r-\mathrm{d}_{1}+\mathrm{d}_{2} \varepsilon\right)}{\mathrm{d}_{2}+a\left(r-\mathrm{d}_{1}+\mathrm{d}_{2} \varepsilon\right)}<d
$$

From (6), we know that 


$$
\frac{\mathrm{d} y}{\mathrm{~d} t} \leq \frac{m c e^{-d \tau}\left(\left(\left(r-\mathrm{d}_{1}\right) / \mathrm{d}_{2}\right)+\varepsilon\right)}{1+a\left(\left(\left(r-\mathrm{d}_{1}\right) / \mathrm{d}_{2}\right)+\varepsilon\right)} y(t-\tau)-\mathrm{d} y(t)=\frac{m c e^{-d \tau}\left(r-\mathrm{d}_{1}+\mathrm{d}_{2} \varepsilon\right)}{d_{2}+a\left(r-\mathrm{d}_{1}+\mathrm{d}_{2} \varepsilon\right)} y(t-\tau)-\mathrm{d} y(t)
$$

for $t>t^{*}$. But for the equation

$$
\frac{\mathrm{d} V}{\mathrm{~d} t}=\frac{m c e^{-d \tau}\left(r-\mathrm{d}_{1}+\mathrm{d}_{2} \varepsilon\right)}{\mathrm{d}_{2}+a\left(r-\mathrm{d}_{1}+\mathrm{d}_{2} \varepsilon\right)} V(t-\tau)-\mathrm{d} V(t)
$$

by (17) and Lemma 3, it follows that $\lim _{t \rightarrow \infty} V(t)=0$. Therefore, an application of the standard comparison argument yields $\lim _{t \rightarrow \infty} y(t)=0$. Then, the limiting equation for $x(t)$ is

$$
\left\{\begin{array}{l}
\frac{\mathrm{d} x}{\mathrm{~d} t}=r x-\mathrm{d}_{1} x-\mathrm{d}_{2} x^{2} \\
x(0)>0 .
\end{array}\right.
$$

Therefore, $\lim _{t \rightarrow \infty} x(t)=\left(\left(r-\mathrm{d}_{1}\right) / \mathrm{d}_{2}\right)$, and $E_{1}$ is globally asymptotically stable in $\widetilde{C}$.

Note also that if (11) holds, then $f(0)=d-\left(\left(m c e^{-d \tau}\left(r-d_{1}\right)\right) /\left(d_{2}+a\left(r-d_{1}\right)\right)\right)<0, \quad$ and $f(+\infty)=\infty$. Hence, $f(\lambda)=0$ has at least one positive root and $E_{1}$ is unstable.

Theorem 1 implies that $E_{1}$ loses its stability to the occurrence of the positive equilibrium $E^{*}$ when (11) holds. The local stability of $E^{*}$ is addressed in the following theorem.

When (11) holds, linearizing system (1) at the positive equilibrium $E^{*}=\left(x^{*}, y^{*}\right)$, we get the characteristic equation as follows:

$$
\lambda^{2}+m_{1} \lambda+m_{2}+e^{-\lambda \tau}\left(m_{3} \lambda+m_{4}\right)=0
$$

where

$$
\begin{aligned}
& m_{1}=\mathrm{d}+\mathrm{d}_{2} x^{*}-\frac{\max ^{*} y^{*}}{\left(1+a x^{*}\right)^{2}}, \\
& m_{2}=\mathrm{d}\left(\mathrm{d}_{2} x^{*}-\frac{\max ^{*} y^{*}}{\left(1+a x^{*}\right)^{2}}\right), \\
& m_{3}=-\mathrm{d}, \\
& m_{4}=\mathrm{d}\left(-\mathrm{d}_{2} x^{*}+\frac{\max ^{*} y^{*}}{\left(1+a x^{*}\right)^{2}}\right) \\
& +\frac{m c e^{-d \tau} y^{*}}{\left(1+a x^{*}\right)^{2}}\left(\frac{r k x^{*}}{\left(1+k y^{*}\right)^{2}}+\frac{m x^{*}}{1+a x^{*}}\right) .
\end{aligned}
$$

When $\tau=0$, (21) yields

$$
\lambda^{2}+\left(m_{1}+m_{3}\right) \lambda+m_{2}+m_{4}=0
$$

It is easy to see that $m_{2}+m_{4}>0$ and $m_{1}+m_{3}=\left(m_{2} / d\right)$. To ensure the local stability of $E^{*}$, we need $m_{2}>0$.

Let $\lambda=i \omega, \omega>0$ is a root of (21); then, (21) implies

$$
\left\{\begin{array}{l}
\omega^{2}-m_{2}=m_{3} \omega \sin (\omega \tau)+m_{4} \cos (\omega \tau) \\
m_{1} \omega=m_{4} \sin (\omega \tau)-m_{3} \omega \cos (\omega \tau)
\end{array}\right.
$$
that

Squaring and adding both equations of (24), it follows

$$
z^{2}+\left(m_{1}^{2}-m_{3}^{2}-2 m_{2}\right) z+m_{2}^{2}-m_{4}^{2}=0,
$$

where $z=\omega^{2}$. A straightforward computation shows that

$$
m_{1}^{2}-m_{3}^{2}-2 m_{2}=\frac{m_{2}^{2}}{\mathrm{~d}^{2}}
$$

Now, we can summarize the above result on the local stability of the positive equilibrium $E^{*}$.

Theorem 2. Assume that (11) holds; then $E^{*}=\left(x^{*}, y^{*}\right)$ exists. Moreover, (i) if $m_{2}>0$ and $m_{2}>m_{4}$, then (21) has no purely imaginary root, and $E^{*}$ is locally asymptotically stable for all $\tau \geq 0$. (ii) If $m_{4}>m_{2}>0$, then (21) has a unique pair of purely imaginary roots $\pm i \omega_{0}$, and $E^{*}$ may lose stability as $\tau$ increases from zero, where $\omega_{0}=\sqrt{z_{0}}$ and $z_{0}=\left(-\left(m_{1}^{2}-m_{3}^{2}\right.\right.$ $\left.\left.-2 m_{2}\right) / 2\right)+\left(\left(\sqrt{\left(m_{1}^{2}-m_{3}^{2}-2 m_{2}\right)^{2}-4\left(m_{2}^{2}-m_{4}^{2}\right)}\right) / 2\right)$ is the unique positive root of (25).

To further investigate the stability of $E^{*}$ under condition (ii) of Theorem 2, we need to check the transversality condition at $\lambda= \pm i \omega_{0}$. Since $x^{*}$ and $y^{*}$ are both time delaydependent, it is hard to discuss the transversality condition, if we suppose that the coefficient in conversing prey into predator $c e^{-d \tau}=c_{0}$ is a constant, independent of delay $\tau$, then we can extend the result in Theorem 2 as follows.

Theorem 3. If $c e^{-d \tau}=c_{0}$ is a constant, $m c_{0}>d a+\left(d d_{2}\right)$ $\left.\left(r-d_{1}\right)\right)$ and $m_{4}>m_{2}>0$, then there exists a sequence of values of $\tau$ :

$$
\tau_{0}<\tau_{1}<\cdots<\tau_{n}<\cdots
$$

such that $E^{*}=\left(x^{*}, y^{*}\right)$ is locally asymptotically stable when $\tau \in\left[0, \tau_{0}\right)$ and unstable when $\tau>\tau_{0}$. Furthermore, system (1) undergoes a Hopf bifurcation at $E_{*}$ when $\tau=\tau_{n}$, $n=0,1,2, \ldots$, where $\tau_{n}$ is defined by (28).

Proof. From (24), we know that $\tau_{n}$ corresponding to $\omega_{0}$ is

$$
\tau_{n}=\frac{1}{\omega_{0}} \arccos \left\{\frac{m_{4}\left(\omega_{0}^{2}-m_{2}\right)-m_{1} m_{3} \omega_{0}^{2}}{m_{3}^{2} \omega_{0}^{2}+m_{4}^{2}}\right\}+\frac{2 n \pi}{\omega_{0}},
$$

$$
n=0,1,2, \ldots
$$


To complete the proof of Theorem 3, we only need to show that

$$
\left.\frac{\mathrm{d}(\operatorname{Re} \lambda)}{\mathrm{d} \tau}\right|_{\tau=\tau_{n}}>0
$$

Differentiating (21) with respect to $\tau$, we have

$$
\begin{gathered}
\left(2 \lambda+m_{1}\right) \frac{\mathrm{d} \lambda}{\mathrm{d} \tau}-\tau e^{-\lambda \tau}\left(m_{3} \lambda+m_{4}\right) \frac{\mathrm{d} \lambda}{\mathrm{d} \tau}-\lambda e^{-\lambda \tau}\left(m_{3} \lambda+m_{4}\right) \\
+m_{3} e^{-\lambda \tau} \frac{\mathrm{d} \lambda}{\mathrm{d} \tau}=0 .
\end{gathered}
$$

$$
\begin{aligned}
\left(\frac{\mathrm{d} \lambda}{\mathrm{d} \tau}\right)^{-1} & =\frac{2 \lambda+m_{1}-\tau e^{-\lambda \tau}\left(m_{3} \lambda+m_{4}\right)+m_{3} e^{-\lambda \tau}}{\lambda e^{-\lambda \tau}\left(m_{3} \lambda+m_{4}\right)} \\
& =\frac{2 \lambda+m_{1}}{\lambda e^{-\lambda \tau}\left(m_{3} \lambda+m_{4}\right)}+\frac{m_{3}}{\lambda\left(m_{3} \lambda+m_{4}\right)}-\frac{\tau}{\lambda} \\
& =\frac{\lambda^{2}-m_{2}}{-\lambda^{2}\left(\lambda^{2}+m_{1} \lambda+m_{2}\right)}-\frac{m_{4}}{\lambda^{2}\left(m_{3} \lambda+m_{4}\right)}-\frac{\tau}{\lambda}
\end{aligned}
$$

(30) and hence,

It follows that

$$
\begin{aligned}
\left.\operatorname{sign}\left\{\frac{\mathrm{d}(\operatorname{Re} \lambda)}{\mathrm{d} \tau}\right\}\right|_{\tau=\tau_{n}} & =\left.\operatorname{sign}\left\{\operatorname{Re}\left(\frac{\mathrm{d} \lambda}{\mathrm{d} \tau}\right)^{-1}\right\}\right|_{\tau=\tau_{n}} \\
& =\operatorname{sign}\left\{\left.\operatorname{Re}\left[\frac{\lambda^{2}-m_{2}}{-\lambda^{2}\left(\lambda^{2}+m_{1} \lambda+m_{2}\right)}\right]\right|_{\tau=\tau_{n}}-\left.\operatorname{Re}\left[\frac{m_{4}}{\lambda^{2}\left(m_{3} \lambda+m_{4}\right)}\right]\right|_{\tau=\tau_{n}}\right\} \\
& =\operatorname{sign}\left\{\left.\operatorname{Re}\left[\frac{\lambda^{2}-m_{2}}{-\lambda^{2}\left(\lambda^{2}+m_{1} \lambda+m_{2}\right)}\right]\right|_{\tau=\tau_{n}}-\left.\operatorname{Re}\left[\frac{m_{4}}{\lambda^{2}\left(m_{3} \lambda+m_{4}\right)}\right]\right|_{\tau=\tau_{n}}\right\} \\
& =\operatorname{sign}\left[\frac{\omega_{0}^{4}-m_{2}^{2}}{\omega_{0}^{2}\left[\left(m_{2}-\omega_{0}^{2}\right)^{2}+m_{1}^{2} \omega_{0}^{2}\right]}+\frac{m_{4}^{2}\left(m_{4}^{2}+m_{3}^{2} \omega_{0}^{2}\right)}{\omega_{0}^{2}}\right] \\
& =\operatorname{sign}\left[\frac{\omega_{0}^{4}-m_{2}^{2}+m_{4}^{2}}{\omega_{0}^{2}\left(m_{4}^{2}+m_{3}^{2} \omega_{0}^{2}\right)}\right] . \\
& F(v, \phi)=\left(\tau_{n}+v\right)\left(F_{1}, F_{2}\right)^{T},
\end{aligned}
$$

Then, we have $\left.(\mathrm{d}(\operatorname{Re} \lambda) / \mathrm{d} \tau)\right|_{\tau=\tau}>0$. Therefore, the transversality condition holds, and the Hopf bifurcation occurs at $\tau=\tau_{n}$.

\section{Properties of Hopf Bifurcations}

In this section, we will study the properties of the Hopf bifurcations by using the normal theory and the center manifold theorem due to Hassard et al. [20].

Let $\quad x_{1}=x-x^{*}, x_{2}=y-y^{*}, \tau=\tau_{n}+v, \bar{x}_{i}(t)=x_{i}(\tau t)$ and dropping the bars for simplification of notations, system (1) is transformed into a functional differential equation in $C=C\left([-1,0], \mathbb{R}^{2}\right)$ as

$$
\dot{x}(t)=L_{v}\left(x_{t}\right)+F\left(v, x_{t}\right)
$$

where $x(t)=x_{1}(t), x_{2}(t)^{T} \in \mathbb{R}^{2}$, and $L_{v}: C \longrightarrow \mathbb{R}^{2}, F: \mathbb{R} \times$ $C \longrightarrow \mathbb{R}^{2}$ are given, respectively, by

$$
\begin{aligned}
L_{v}(\phi)= & \left(\tau_{n}+v\right)\left(\begin{array}{cc}
a_{11} & a_{12} \\
0 & a_{22}
\end{array}\right)\left(\begin{array}{l}
\phi_{1}(0) \\
\phi_{2}(0)
\end{array}\right) \\
& +\left(\tau_{n}+v\right)\left(\begin{array}{cc}
0 & 0 \\
b_{21} & b_{22}
\end{array}\right)\left(\begin{array}{l}
\phi_{1}(-1) \\
\phi_{2}(-1)
\end{array}\right),
\end{aligned}
$$

and where

$$
\begin{aligned}
a_{11}= & \frac{r}{1+k y^{*}}-d_{1}-2 \mathrm{~d}_{2} x^{*}-\frac{m y^{*}}{\left(1+a x^{*}\right)^{2}}, \\
a_{12}= & -\frac{k r x^{*}}{\left(1+k y^{*}\right)^{2}}-\frac{m x^{*}}{1+a x^{*}}, \\
a_{22}= & -d, \\
b_{21}= & \frac{c_{0} m y^{*}}{\left(1+a x^{*}\right)^{2}}, \\
b_{22}= & \frac{c_{0} m x^{*}}{1+a x^{*}}, \\
F_{1}= & K_{11} \phi_{1}^{2}(0)+K_{12} \phi_{1}(0) \phi_{2}(0)+K_{13} \phi_{2}^{2}(0)+K_{14} \phi_{1}^{3}(0) \\
& +K_{15} \phi_{1}^{2}(0) \phi_{2}(0)+K_{16} \phi_{1}(0) \phi_{2}^{2}(0)+K_{17} \phi_{2}^{3}(0)+\cdots \\
F_{2}= & K_{21} \phi_{1}^{2}(-1)+K_{22} \phi_{1}(-1) \phi_{2}(-1) \\
& +K_{23} \phi_{1}^{3}(-1)+K_{24} \phi_{1}^{2}(-1) \phi_{2}(-1)+\cdots,
\end{aligned}
$$




$$
\begin{aligned}
& K_{11}=\frac{m a y^{*}}{\left(1+a x^{*}\right)^{3}}-d_{2}, \\
& K_{12}=-\frac{k r}{\left(1+k y^{*}\right)^{2}}-\frac{m}{\left(1+a x^{*}\right)^{2}}, \\
& K_{13}=\frac{k^{2} r x^{*}}{\left(1+k y^{*}\right)^{3}}, \\
& K_{14}=-\frac{m a^{2} y^{*}}{\left(1+a x^{*}\right)^{4}}, \\
& K_{15}=\frac{m a}{\left(1+a x^{*}\right)^{3}}, \\
& K_{16}=\frac{k^{2} r}{\left(1+k y^{*}\right)^{3}}, \\
& K_{17}=-\frac{k^{3} r x^{*}}{\left(1+k y^{*}\right)^{4}}, \\
& K_{24}=-\frac{c_{0} m a y^{*}}{\left(1+a x^{*}\right)^{3}} . \\
& K_{22}=\frac{c_{0} m a^{2} y^{*}}{\left(1+a x^{*}\right)^{3}}, \\
& \left(1+a x^{*}\right)^{2}
\end{aligned}
$$

By the Riesz representation theorem, there exists a function $\eta(\theta, v)$ of bounded variation for $\theta \in[-1,0]$, such that

$$
L_{v}(\phi)=\int_{-1}^{0} \mathrm{~d} \eta(\theta, v) \phi(\theta), \quad \text { for } \phi \in C .
$$

In fact, we can choose

$$
\begin{aligned}
\eta(\theta, v)= & \left(\tau_{n}+v\right)\left(\begin{array}{cc}
a_{11} & a_{12} \\
0 & a_{22}
\end{array}\right) \delta(\theta) \\
& -\left(\tau_{n}+v\right)\left(\begin{array}{cc}
0 & 0 \\
b_{21} & b_{22}
\end{array}\right) \delta(\theta+1),
\end{aligned}
$$

where $\delta$ denotes the Dirac delta function. For $\phi \in C^{1}\left([-1,0], \mathbb{R}^{2}\right)$, definex

$$
A(v) \phi=\left\{\begin{array}{ll}
\frac{\mathrm{d} \phi(\theta)}{\mathrm{d} \theta}, & \theta \in[-1,0), \\
\int_{-1}^{0} \mathrm{~d} \eta(s, v) \phi(s), & \theta=0,
\end{array} \quad R(v)(\phi)= \begin{cases}0, & \theta \in[-1,0), \\
F(v, \phi), & \theta=0 .\end{cases}\right.
$$

Then, system (1) is equivalent to

$$
\dot{x}_{t}=A(v) x_{t}+R(v) x_{t}
$$

where $\quad x_{t}(\theta)=x(t+\theta) \quad$ for $\quad \theta \in[-1,0]$. For $\psi \in C^{1}\left([0,1],\left(\mathbb{R}^{2}\right)^{*}\right)$, define

$$
A^{*} \psi(s)= \begin{cases}-\frac{\mathrm{d} \psi(s)}{\mathrm{d} s}, & s \in(0,1], \\ \int_{-1}^{0} \mathrm{~d} \eta^{T}(t, 0) \psi(-t), & s=0,\end{cases}
$$

and a bilinear inner product

$$
\langle\psi(s), \phi(\theta)\rangle=\bar{\psi}(0) \phi(0)-\int_{-1}^{0} \int_{\xi=0}^{\theta} \bar{\psi}(\xi-\theta) \mathrm{d} \eta(\theta) \phi(\xi) \mathrm{d} \xi,
$$

where $\eta(\theta)=\eta(\theta, 0)$. Then, $A(0)$ and $A^{*}$ are adjoint operators. By the discussion in Section 3 , we know that $\pm i \omega_{0} \tau_{n}$ are eigenvalues of $A(0)$. Hence, they are also eigenvalues of $A^{*}$. We first need to compute the eigenvectors of $A(0)$ and $A^{*}$ corresponding to $i \omega_{0} \tau_{n}$ and $-i \omega_{0} \tau_{n}$, respectively.

Suppose $q(\theta)=(1, \alpha)^{T} e^{i \omega_{0} \tau_{n} \theta}$ is the eigenvector of $A(0)$ corresponding to $i \omega_{0} \tau_{n}$; then $A(0) q(\theta)=i \omega_{0} \tau_{n} q(\theta)$. Then, from the definition of $A(0),(34),(37)$, and (38), we have

$$
\alpha=\frac{i \omega_{0}-a_{11}}{a_{12}} \text {. }
$$

Similarly, we can obtain the eigenvector $q^{*}(s)=D\left(1, \alpha^{*}\right) e^{i \omega_{0} \tau_{n} s}$ of $A^{*}$ corresponding to $-i \omega_{0} \tau_{n}$, where

$$
\alpha^{*}=-\frac{i \omega_{0}+a_{11}}{b_{21} e^{i \omega_{0} \tau_{n}}}
$$


Choosing $D$ as $D=\left\{1+\bar{\alpha} \alpha^{*}+\alpha^{*} \tau_{n} e^{i \omega_{0} \tau_{n}}\left(b_{21}+\bar{\alpha} b_{22}\right)\right\}^{-1}$, then by (42), we see $\left\langle q^{*}(s), q(\theta)\right\rangle=1$.

Now, we will use the algorithms given in [20] and using a computation process similar to that in [21-23], we get the coefficients used in determining the qualities of bifurcating periodic solutions:

$$
\begin{aligned}
g_{20}= & 2 \tau_{n} \bar{D}\left[K_{11}+\alpha K_{12}+\alpha^{2} K_{13}+\bar{\alpha}^{*}\left(K_{21}+\alpha K_{22}\right) e^{-2 i \omega_{0} \tau_{n}}\right], \\
g_{11}= & 2 \tau_{n} \bar{D}\left[K_{11}+K_{12} \operatorname{Re}\{\alpha\}+\alpha \bar{\alpha} K_{13}+\bar{\alpha}^{*}\left(K_{21}+K_{22} \operatorname{Re}\{\alpha\}\right)\right], \\
g_{02}= & 2 \tau_{n} \bar{D}\left[K_{11}+\bar{\alpha} K_{12}+\bar{\alpha}^{2} K_{13}+\bar{\alpha}^{*}\left(K_{21}+\bar{\alpha} K_{22}\right) e^{2 i \omega_{0} \tau_{n}}\right], \\
g_{21}= & 2 \tau_{n} \bar{D}\left\{\left(W_{20}^{(1)}(0)+2 W_{11}^{(1)}(0)\right) K_{11}\right. \\
& +\left(W_{11}^{(2)}(0)+\frac{1}{2} W_{20}^{(2)}(0)+\frac{\bar{\alpha}}{2} W_{20}^{(1)}(0)\right) K_{12} \\
& +\left(2 \alpha W_{11}^{(2)}(0)+\bar{\alpha} W_{20}^{(2)}(0)\right) K_{13}+3 K_{14}+(2 \alpha+\bar{\alpha}) K_{15}+\alpha(2 \bar{\alpha}+\alpha) K_{16} \\
& +3 \alpha^{2} \bar{\alpha} K 17+\bar{\alpha}^{*}\left(2 W_{11}^{(1)}(-1) e^{-i \omega_{0} \tau_{n}}+W_{20}^{(1)}(-1) e^{i \omega_{0} \tau_{n}}\right) K_{21} \\
& +\left(\left(W_{11}^{(2)}(-1)+\alpha W_{11}^{(1)}(-1)\right) e^{-i \omega_{0} \tau_{n}}+\frac{1}{2}\left(W_{20}^{(2)}(-1)+\bar{\alpha} W_{20}^{(1)}(-1)\right) e^{i \omega_{0} \tau_{n}}\right) K_{22} \\
& \left.\left.+\left(3 K_{23}+(\bar{\alpha}+2 \alpha) K_{24}\right) e^{-i \omega_{0} \tau_{n}}\right\}\right),
\end{aligned}
$$

where

$$
\begin{aligned}
W_{20}(\theta) & =\frac{i g_{20}}{\omega_{0} \tau_{n}} q(0) e^{i \omega_{0} \tau_{n} \theta}+\frac{i \bar{g}_{02}}{3 \omega_{0} \tau_{n}} \bar{q}(0) e^{-i \omega_{0} \tau_{n} \theta}+E_{1} e^{2 i \omega_{0} \tau_{n} \theta}, \\
W_{11}(\theta) & =-\frac{i g_{11}}{\omega_{0} \tau_{n}} q(0) e^{i \omega_{0} \tau_{n} \theta}+\frac{i \bar{g}_{11}}{\omega_{0} \tau_{n}} \bar{q}(0) e^{-i \omega_{0} \tau_{n} \theta}+E_{2}, \\
E_{1}^{(1)} & =\frac{M_{11}}{M_{1}}, \\
E_{1}^{(2)} & =\frac{M_{12}}{M_{1}}, \\
E_{2}^{(1)} & =\frac{M_{21}}{M_{2}}, \\
E_{2}^{(2)} & =\frac{M_{22}}{M_{2}}, \\
M_{1} & =\left|\begin{array}{cc}
2 i \omega_{0}-a_{11} & -a_{12} \\
-b_{21} e^{-2 i \omega_{0} \tau_{n}} & 2 i \omega_{0}-a_{22}-b_{22} e^{-2 i \omega_{0} \tau_{n}}
\end{array}\right|, \\
M_{11} & =2\left|\begin{array}{cc}
K_{11}+\alpha K_{12}+\alpha^{2} K_{13} & -a_{12} \\
K_{21}+\alpha K_{22} & 2 i \omega_{0}-a_{22}-b_{22} e^{-2 i \omega_{0} \tau_{n}}
\end{array}\right|, \\
M_{12} & =2\left|\begin{array}{cc}
2 i \omega_{0}-a_{11} & K_{11}+\alpha K_{12}+\alpha^{2} K_{13} \\
-b_{21} e^{-2 i \omega_{0} \tau_{n}} & K_{21}+\alpha K_{22}
\end{array}\right|,
\end{aligned}
$$

$$
\begin{aligned}
M_{2} & =\left|\begin{array}{cc}
-a_{11} & -a_{12} \\
-b_{21} & -a_{22}-b_{22}
\end{array}\right|, \\
M_{21} & =2\left|\begin{array}{cc}
K_{11}+K_{12} \operatorname{Re}\{\alpha\}+\alpha \bar{\alpha} K_{13} & -a_{12} \\
K_{21}+K_{22} \operatorname{Re}\{\alpha\} & -a_{22}-b_{22}
\end{array}\right|, \\
M_{22} & =2\left|\begin{array}{cc}
-a_{11} & K_{11}+K_{12} \operatorname{Re}\{\alpha\}+\alpha \bar{\alpha} K_{13} \\
-b_{21} & K_{21}+K_{22} \operatorname{Re}\{\alpha\}
\end{array}\right| .
\end{aligned}
$$

Thus, we can determine $W_{20}(\theta)$ and $W_{11}(\theta)$. Furthermore, we can compute $g_{21}$ by (45). Then, we can compute the following values:

$$
\begin{aligned}
c_{1}(0) & =\frac{i}{2 \omega_{0} \tau_{n}}\left(g_{20} g_{11}-2\left|g_{11}\right|^{2}-\frac{\left|g_{02}\right|^{2}}{3}\right)+\frac{g_{21}}{2}, \\
\mu_{2} & =-\frac{\operatorname{Re}\left\{c_{1}(0)\right\}}{\operatorname{Re}\left\{\lambda^{\prime}\left(\tau_{n}\right)\right\}}, \\
T_{2} & =-\frac{\operatorname{Im}\left\{c_{1}(0)\right\}+\mu_{2} \operatorname{Im}\left\{\lambda^{\prime}\left(\tau_{n}\right)\right\}}{\omega_{0} \tau_{n}}, \\
\beta_{2} & =2 \operatorname{Re}\left\{c_{1}(0)\right\} .
\end{aligned}
$$

The properties of bifurcating periodic solution in the center manifold at the critical value $\tau_{n}$ are determined by the values in (47). From [20], we know that $\mu_{2}$ determines the directions of the Hopf bifurcation: if $\mu_{2}>0\left(\mu_{2}<0\right)$, then the Hopf bifurcation is supercritical (subcritical); $\beta_{2}$ determines the stability of the bifurcating periodic solutions: the 
bifurcating periodic solutions are stable (unstable) if $\beta_{2}<0\left(\beta_{2}>0\right)$; and $T_{2}$ determines the period of the bifurcating periodic solutions: the period increase (decrease) if $T_{2}>0\left(T_{2}<0\right)$.

\section{Permanence}

In order to prove the uniform persistence of system (1), we need the uniform persistence theorem for infinite dimensional systems from [24]. Let $X$ be a complete metric space. Suppose that $X^{0}$ is open and dense in $X$, with $X^{0} \cup X_{0}=X$, $X^{0} \cap X_{0}=\varnothing$. Assume that $T(t)$ is a $C^{0}$-semigroup on $X$ satisfying

$$
\begin{aligned}
& T(t): X^{0} \longrightarrow X^{0}, \\
& T(t): X_{0} \longrightarrow X_{0} .
\end{aligned}
$$

Let $T_{b}(t)=\left.T(t)\right|_{X_{0}}$ and let $A_{b}$ be the global attractor for $T_{b}(t)$. Assume further that

(i) There is a $t_{0} \geq 0$ such that $T(t)$ is compact for $t>t_{0}$;

(ii) $T(t)$ is point dissipative in $X$;

(iii) $\tilde{A}_{b}=\cup_{x \in A_{b}} \omega(x)$ is isolated and has an acyclic covering $M$, where $M=\cup_{i=1}^{k} M_{k}$;

(iv) $W^{s}\left(M_{i}\right) \cap X^{0}=\varnothing$ for $i=1,2, \ldots, k$.

Then, $X_{0}$ is a uniform repeller with respect to $X^{0}$, i.e., there is an $\epsilon>0$ such that for any $x \in X^{0}$, $\liminf _{t \rightarrow+\infty} \mathrm{d}\left(T(t) x, X_{0}\right) \geq \mathcal{E}$, where $d$ is the distance of $T(t) x$ from $X_{0}$.

Now, we consider the persistence of system (1).

Theorem 4. Let (11) hold; then there exists an $\varepsilon>0$ such that every solution $x(t), y(t)$ of system (1) with initial conditions $\phi_{1}(\theta) \geq 0, \phi_{2}(\theta) \geq 0$ and $\phi_{1}\left(\theta_{0}\right) \phi_{2}\left(\theta_{0}\right) \neq 0$ for some $\theta_{0} \in[-\tau, 0]$ satisfies

$$
\begin{aligned}
& \liminf _{t \longrightarrow \infty} x(t) \geq \varepsilon, \\
& \liminf _{t \longrightarrow \infty} y(t) \geq \varepsilon .
\end{aligned}
$$

Proof. Let

$$
\begin{aligned}
X & =C\left([-\tau, 0], R_{+}^{2}\right), \\
X^{0} & =\left\{\left(\phi_{1}, \phi_{2}\right) \in X \mid \phi_{1}\left(\theta^{*}\right) \phi_{2}\left(\theta^{*}\right)>0 \text { for some } \theta^{*} \in[-\tau, 0]\right\}, \\
X_{0} & =X \backslash X^{0}=\left\{\left(\phi_{1}, \phi_{2}\right) \in X \mid \phi_{1}(\theta) \phi_{2}(\theta) \equiv 0 \text { for all } \theta \in[-\tau, 0]\right\} .
\end{aligned}
$$

It suffices to show that there exists an $\varepsilon>0$ such that for any solution $u_{t}$ of system (1) initiating from $X^{0}, \liminf \operatorname{in}_{t \rightarrow \infty} \mathrm{d}\left(u_{t}, X_{0}\right) \geq \varepsilon$. To this end, we verify below that the conditions of the above uniform persistence theorem are satisfied. Firstly, we show $X^{0}$ is positively invariant. By (1), we have

$$
x(t)=x\left(\theta^{*}\right) \exp \left(\int_{\theta^{*}}^{t}\left[\frac{r}{1+k y(\rho)}-\mathrm{d}_{1}-\mathrm{d}_{2} x(\rho)-\frac{m y(\rho)}{1+a x(\rho)}\right] \mathrm{d} \rho\right),
$$

and

$$
y(t)=\left[y(0)+\int_{0}^{t} \frac{m x(\rho-\tau) y(\rho-\tau) e^{-\mathrm{d}(\tau-\rho)}}{1+a x(\rho-\tau)} \mathrm{d} \rho\right] e^{-\mathrm{d} t} .
$$

Then, $x(t)>0$ and $y(t)>0$ for all $t>0$ if $\Phi=\left(\phi_{1}, \phi_{2}\right) \in X^{0}$. This implies that $X^{0}$ is positively invariant.

Similarly, we can show that $X_{0}$ is positively invariant, and condition (48) is satisfied. We have verified the point dissipativeness of the semiflow of system (1) in 2.

Denote the $\omega$-limit set of the solution of system (1) starting in $\left(\phi_{1}, \phi_{2}\right) \in X$ by $\omega\left(\phi_{1}, \phi_{2}\right)$. Let

$$
\Omega=\cup\left\{\omega\left(\phi_{1}, \phi_{2}\right) \mid\left(\phi_{1}, \phi_{2}\right) \in X_{0}\right\} .
$$

For any given $\left(\phi_{1}, \phi_{2}\right) \in X_{0}$, we have $\phi_{1}(\theta) \phi_{2}(\theta) \equiv 0$ for all $\theta \in[-\tau, 0]$. If $\phi_{1}(\theta)=0$ for some $\theta \in[-\tau, 0]$, then $x(t) \equiv 0$ for all $t \geq 0$, and $y(t) \longrightarrow 0$ as $t \longrightarrow \infty$. If $\phi_{2}(\hat{\theta})=0$ for some $\hat{\theta} \in[-\tau, 0]$, we have the following two cases.

Case 1. $y(0)=0$. Then from (52), we have $y(t) \equiv 0$ for all $t \geq 0$, and $\dot{x}=r x-d_{1} x-d_{2} x^{2}, \quad$ it follows that $x(t) \longrightarrow \operatorname{0or} x(t) \longrightarrow\left(r-\mathrm{d}_{1} / \mathrm{d}_{2}\right)$ as $t \longrightarrow \infty$.

Case 2. $y(0)>0$. Then we must have $x(0)=0$; from the first equation of (1), we have $x(t) \equiv 0$ for all $t \geq 0$; again from the second equation of (1), we have $y(t) \longrightarrow 0$ as $t \longrightarrow \infty$.

Finally, we see that for any given $\left(\phi_{1}, \phi_{2}\right) \in X_{0},(x(t), y(t)) \longrightarrow E_{0} \quad$ as $\quad t \longrightarrow \infty$, or $x(t), y(t) \longrightarrow E_{1}$ as $t \longrightarrow \infty$. Thus, $\Omega=\left\{E_{0}, E_{1}\right\}$. If invariant sets $E_{0}$ and $E_{1}$ are isolated, then $\Omega$ is isolated and is an acyclic covering. The isolated invariance of $E_{0}$ and $E_{1}$ will follow from the following proof.

We now show that

$$
\begin{aligned}
& W^{s}\left(E_{0}\right) \cap X^{0}=\varnothing, \\
& W^{s}\left(E_{1}\right) \cap X^{0}=\varnothing,
\end{aligned}
$$

where $W^{s}\left(E_{0}\right)\left(W^{s}\left(E_{1}\right)\right)$ denotes the stable manifold of $E_{0}\left(E_{1}\right)$. Suppose $W^{s}\left(E_{0}\right) \cap X^{0} \neq \varnothing$; then there exists a solution $(x(t), y(t)) \in X^{0}, t \geq 0$, of (1) with initial conditions in $X^{0}$, such that

$$
\begin{aligned}
& \lim _{t \longrightarrow \infty} x(t)=0, \\
& \lim _{t \longrightarrow \infty} y(t)=0 .
\end{aligned}
$$

Since $r>d_{1}$, we can choose sufficiently small $\eta_{1}>0$ so that

$$
\frac{r}{1+k \eta_{1}}-d_{1}-m \eta_{1}>0
$$

Lett $_{0}>0$ sufficiently large such that 


$$
0<y(t)<\eta_{1}, \quad t>t_{0} .
$$

Then, for $t>t_{0}$, we have

$$
\left\{\begin{array}{l}
\frac{\mathrm{d} x}{\mathrm{~d} t} \geq \frac{r}{1+k \eta_{1}} x-\mathrm{d}_{1} x-\mathrm{d}_{2} x^{2}-m \eta_{1} x, \\
x\left(t_{0}\right)>0 .
\end{array}\right.
$$

Consider the following equation:

$$
\left\{\begin{array}{l}
\frac{\mathrm{d} W}{\mathrm{~d} t}=\frac{r}{1+k \eta_{1}} W-\mathrm{d}_{1} W-\mathrm{d}_{2} W^{2}-m \eta_{1} W, \\
W\left(t_{0}\right)=x\left(t_{0}\right)>0 .
\end{array}\right.
$$

By the comparison principle, we have $x(t) \geq W(t)$ for all $t \geq t_{0}$. It is easy to see that (59) has a positive equilibrium $W^{*}=\left(\left(\left(r / 1+k \eta_{1}\right)-\mathrm{d}_{1}-m \eta_{1}\right) / \mathrm{d}_{2}\right)$, which is globally attractive. Note that $x(t) \geq W(t)$ for all $t \geq t_{0}$ and $\lim _{t \rightarrow \infty} W(t)=W^{*}$; this is a contradiction to (55).

Suppose $W^{s}\left(E_{1}\right) \cap X^{0} \neq \varnothing$; then there exists a solution $x(t), y(t) \in X^{0}, t \geq 0$, of (1) with initial conditions in $X^{0}$, such that

$$
\begin{aligned}
& \lim _{t \longrightarrow \infty} x(t)=\frac{r-d_{1}}{d_{2}}, \\
& \lim _{t \longrightarrow \infty} y(t)=0 .
\end{aligned}
$$

Then, for any $\left(\left(r-d_{1}\right) / d_{2}\right)>\eta_{2}>0$, by $(60)$, there exists a $t_{1}>0$ such that

$$
\frac{r-\mathrm{d}_{1}}{\mathrm{~d}_{2}}-\eta_{2}<x(t)<\frac{r-\mathrm{d}_{1}}{\mathrm{~d}_{2}}+\eta_{2}, \quad \text { for all } t>t_{1}-\tau .
$$

Hence, by (1),

$$
\begin{aligned}
\frac{\mathrm{d} y(t)}{\mathrm{d} t} & \geq \frac{m c e^{-\mathrm{d} \tau}\left(\mathrm{d}_{2}-\eta_{2}\right)}{1+a\left(\left(\left(r-\mathrm{d}_{1}\right) / \mathrm{d}_{2}\right)-\eta_{2}\right)} y(t-\tau)-\mathrm{d} y(t), \\
& =\frac{m c e^{-d \tau}\left(r-\mathrm{d}_{1}-\mathrm{d}_{2} \eta_{2}\right)}{\mathrm{d}_{2}+a\left(r-\mathrm{d}_{1}-\mathrm{d}_{2} \eta_{2}\right)} y(t-\tau)-\mathrm{d} y(t), \quad t>t_{1} .
\end{aligned}
$$

Since (11) holds, we can choose $\eta_{2}>0$ sufficiently small so that $\left(\left(m c e^{-d \tau}\left(r-d_{1}-\mathrm{d}_{2} \eta_{2}\right)\right) /\left(\mathrm{d}_{2}+a\left(r-\mathrm{d}_{1}-\right.\right.\right.$ $\left.\left.\left.\mathrm{d}_{2} \eta_{2}\right)\right)\right)>\mathrm{d}$. Then, by Lemma 3 , the solution of the linear delay differential equation $\dot{u}(t)=\left(\left(m c e^{-\mathrm{d} \tau}\left(r-\mathrm{d}_{1}-\mathrm{d}_{2} \eta_{2}\right)\right) /\right.$ $\left.\left(\mathrm{d}_{2}+a\left(r-\mathrm{d}_{1}-\mathrm{d}_{2} \eta_{2}\right)\right)\right) u(t-\tau)-\mathrm{d} u(t)$ with positive initial condition must converge to infinity as $t \longrightarrow \infty$. Therefore, by the comparison principle, we have $y(t) \longrightarrow \infty$ as $t \longrightarrow \infty$, which is a contradiction to (60). Thus, (54) holds. This completes the proof.

In Lemma 2, we have shown that the solutions of system (1) are ultimately bounded; together with Theorem 4, we now give the main result in this section.

Theorem 5. System 1 is permanent provided 11 holds and the initial conditions are shown in Theorem 4.

\section{Numerical Simulations}

In order to investigate the role of time delay and fear in our model (1), we perform some numerical simulations to illustrate the analytical results observed in the previous sections. All the simulations are carried out in MATLAB.

First, we choose the parameter values as

$$
\begin{aligned}
r & =0.1, \\
k & =0.3, \\
d_{1} & =0.01, \\
d_{2} & =0.01, \\
m & =0.5, \\
c & =0.4, \\
d & =0.22, \\
a & =0.6, \\
\tau & =0.1,
\end{aligned}
$$

where most of the parameters are taken from [12].

For the above set of parameter values, in Figure 1, we observe that the system shows a limit cycle around the interior equilibrium $E^{*}$. If we further increase the value of $\tau$, for $\tau=0.4$, we see that the solutions approach the coexistence equilibrium; see Figure 2 .

To observe the long-term behavior for a range of values of $\tau$, in Figure 3, we draw the bifurcation diagram with respect to $\tau$. From Figure 3, we can see that as we increase the value of $\tau$, the prey and predator populations show oscillatory coexistence for the small values of $\tau$; then, both populations show stable coexistence. This indicates that increasing delay $\tau$ may induce a transition from the state where the populations of the prey and predator oscillate 


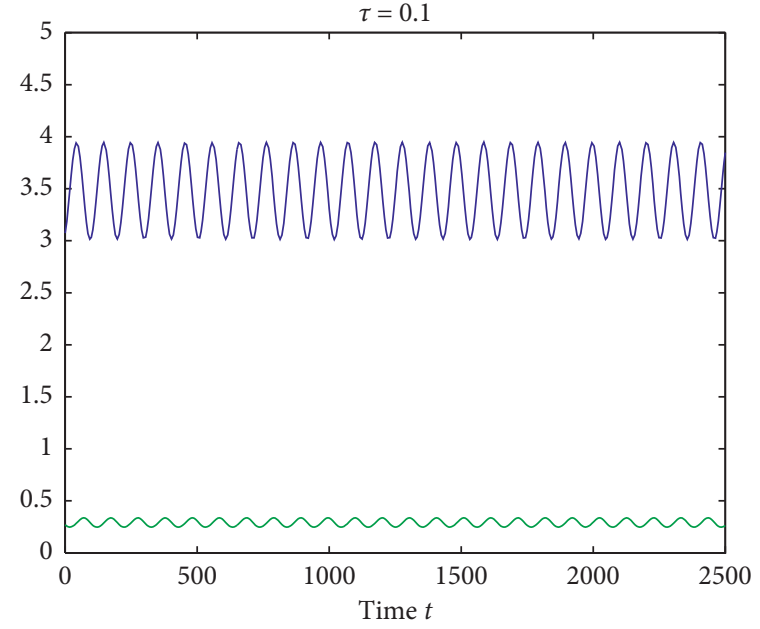

(a)

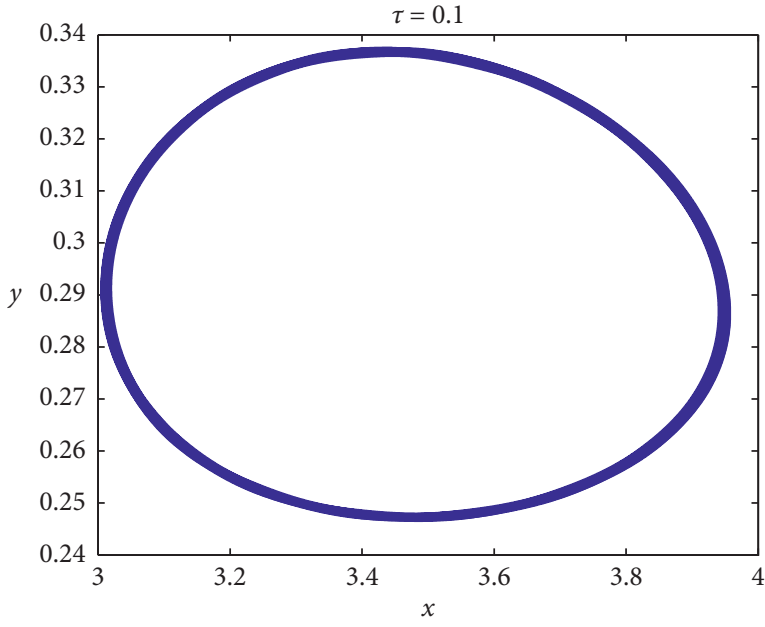

(b)

Figure 1: The figure shows that the dynamics of system (1) of prey and predator populations. (a) represents the stable and periodic oscillations around the interior equilibrium $E^{*}$ and the phase plane diagram. (b) represents the stable limit cycle around $E^{*}$ when $\tau=0.1$. The parameter values are given in (63).

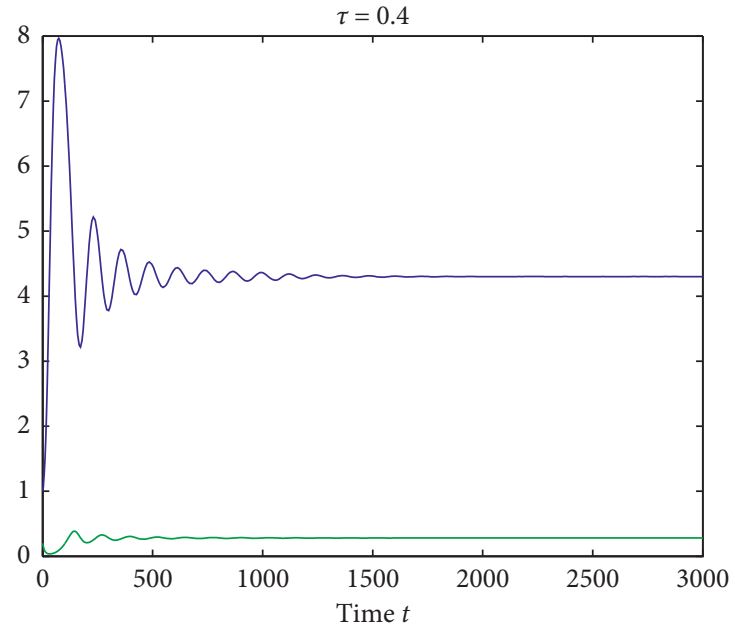

(a)

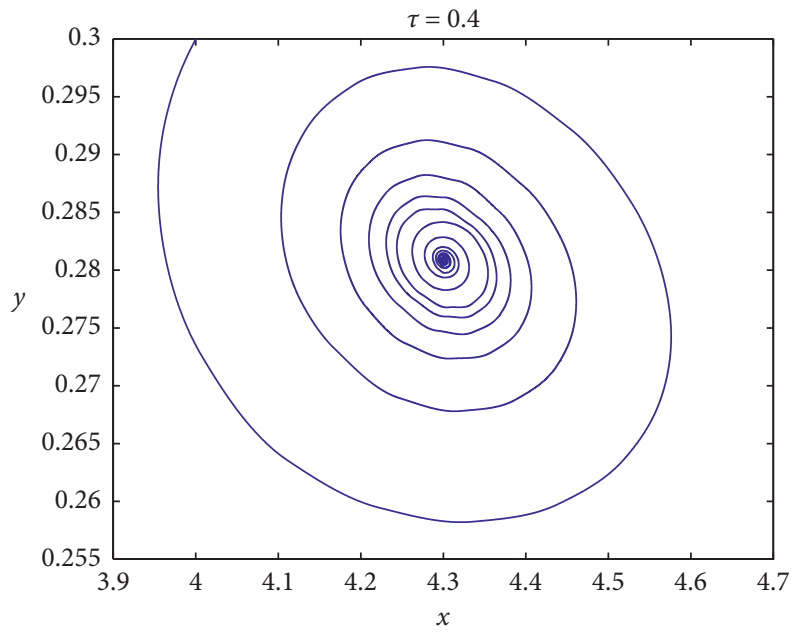

(b)

Figure 2: The figure shows that the dynamics of the model system (1) of prey and predator populations. (a) represents the coexistence equilibrium $E^{*}$ that is locally asymptotically stable and (b) represents the phase-portrait of $E^{*}$ that is locally asymptotically stable when $\tau=0.4$. The other parameter values are the same as in Figure 1 .

periodically to a stability situation. And also, we note that the prey population increases, while the predator population decreases as we gradually increase $\tau$, and finally the predator population goes to extinction; the system becomes stable around the semitrivial equilibrium $E_{1}$.

To investigate the impact of fear when the system is unstable around the positive equilibrium, we fix $\tau=0.2$. We draw the bifurcation diagram considering $k$ as a bifurcation parameter. Figure 4 shows that as we increase the level of the fear effect in prey, the coexistence of prey-predator changes from stable periodic oscillations to stable equilibrium. Figure 4 indicates that the prey population tends to a steady state. The fear effect will not affect the prey population over the long term. Biologically, after a certain level of fear with prey population, the fear has no effect due to physiological impact when the prey populations are habituated with fear from predator species, while the predator population eventually will decrease with increasing $k$.

If we choose parameter values as those in Figure 1 and let $m$ vary in the interval $[0.3,0.65]$, Figure 5 gives the 


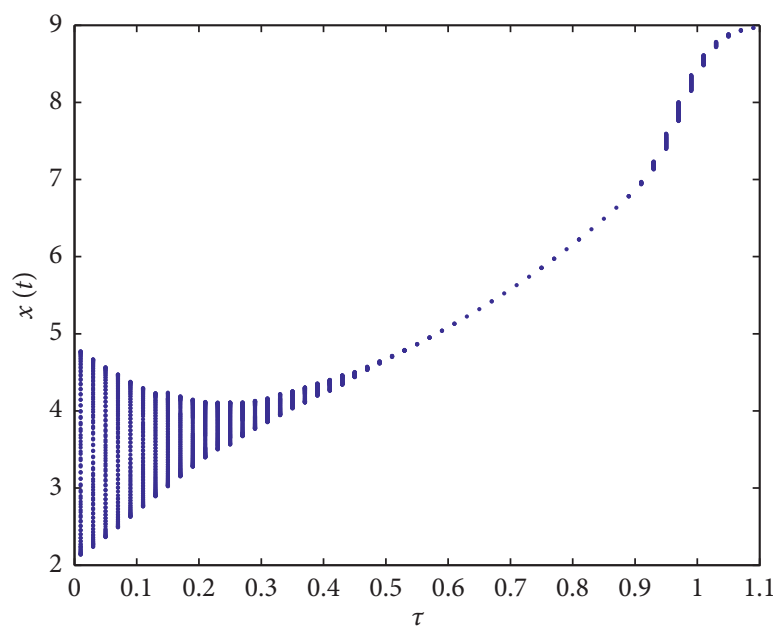

(a)

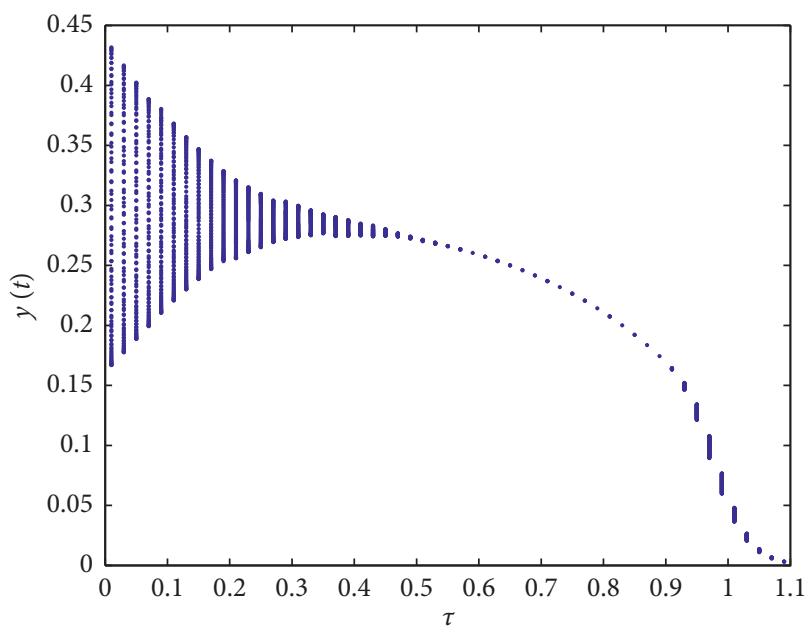

(b)

Figure 3: The figure shows the bifurcation diagrams of system (1) of prey and predator populations with respect to the parameter $\tau$, which indicates a transition from the state where the populations of the prey and predator oscillate periodically to a stability situation. The other parameters are given in (63).

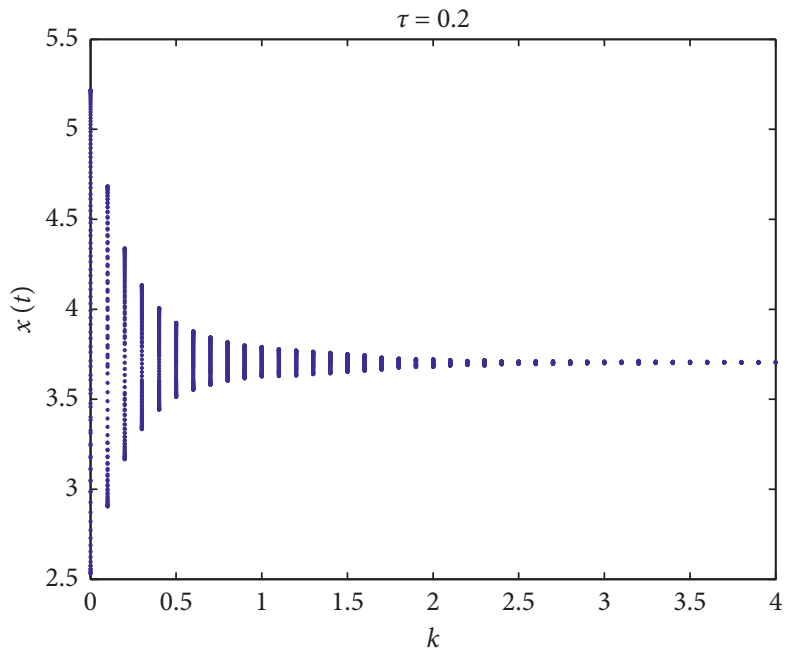

(a)

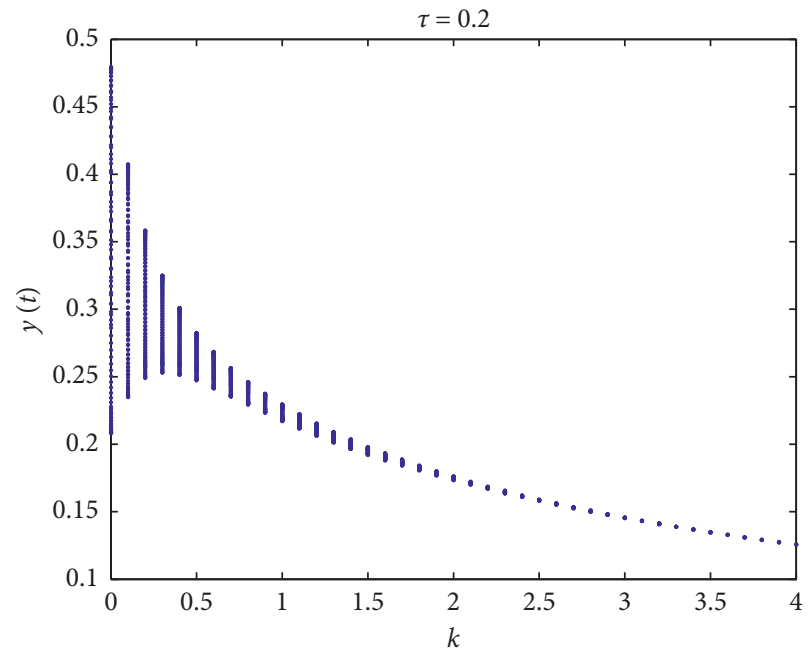

(b)

Figure 4: The figure shows the bifurcation diagrams of system (1) of prey and predator populations with respect to the parameter $k$, which indicates that high levels of fear can stabilize the predator-prey system by excluding the existence of periodic solutions, where $\tau=0.2$ and other parameters are given in (63).

bifurcation diagram. From this figure, we can see that the predator population can survive only for the large values of $m$. If the value of the predation rate is low, then the predator population cannot survive. As we increase the value of $m$, the coexistence of prey-predator changes at stable equilibrium to stable oscillatory coexistence.

To investigate the properties of the bifurcating periodic solution, we neglect the delay-dependent factor $e^{-d \tau}$; that is, we consider the following system:

$$
\left\{\begin{array}{l}
\frac{\mathrm{d} x}{\mathrm{~d} t}=\frac{r x}{1+k y}-\mathrm{d}_{1} x-\mathrm{d}_{2} x^{2}-\frac{m x y}{1+a x} \\
\frac{\mathrm{d} y}{\mathrm{~d} t}=\frac{m c_{0} x(t-\tau) y(t-\tau)}{1+a x(t-\tau)}-\mathrm{d} y
\end{array}\right.
$$

We use the following parameter values: 


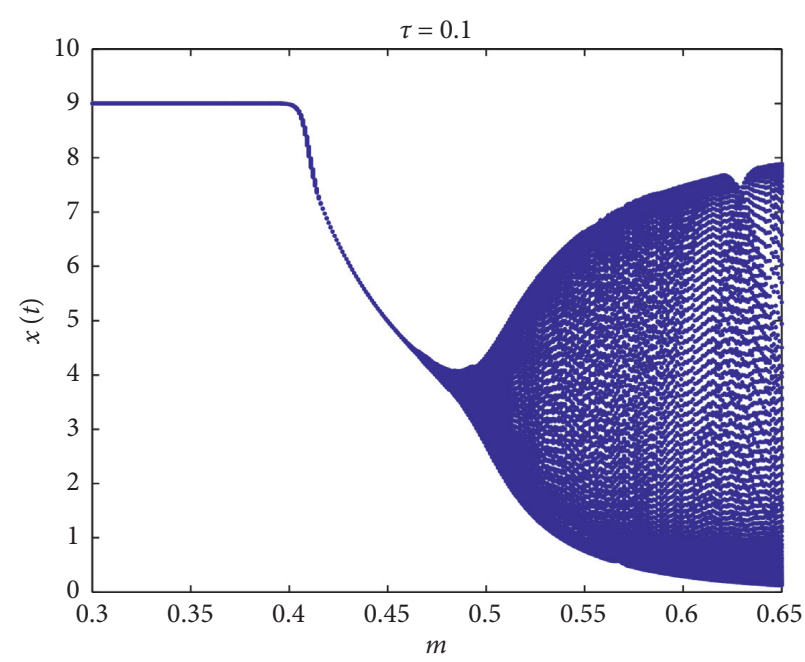

(a)

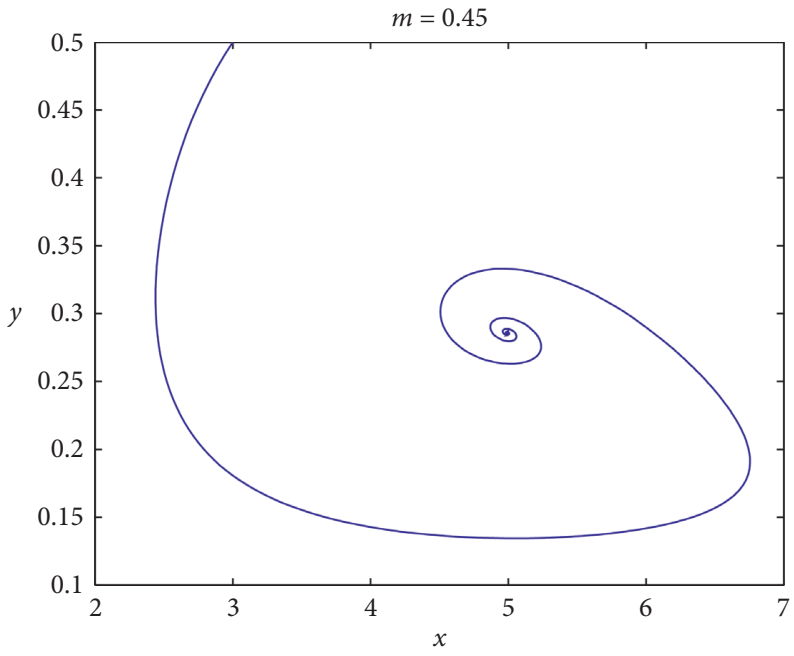

(c)

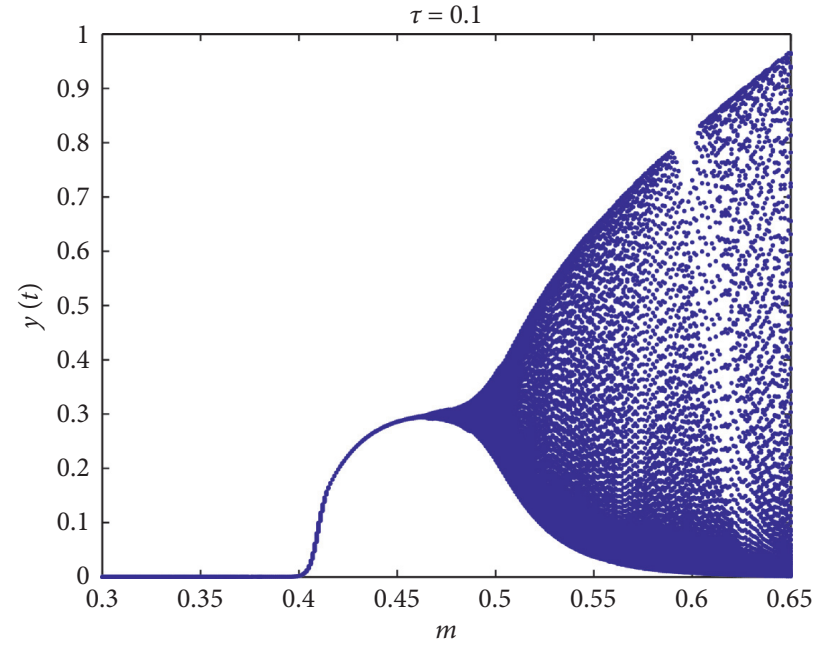

(b)

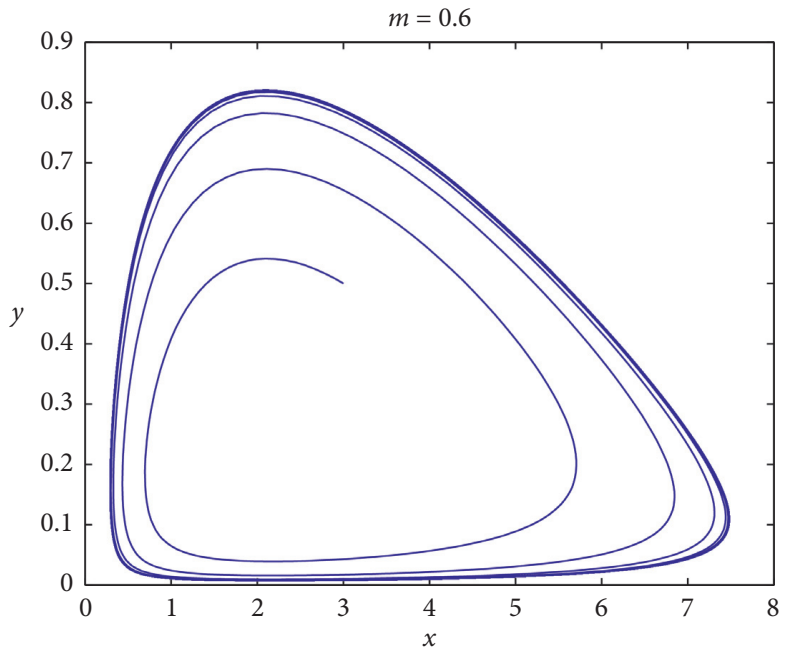

(d)

Figure 5: (a) and (b) are the bifurcation diagrams of prey and predators with respect to parameter $m$, respectively, which indicate the emergence of Hopf bifurcations as $k$ increases. The parameters are given in (63). In (c), $\tau=0.1, m=0.45$, and the positive equilibrium is asymptotically stable. In (d), $\tau=0.1, m=0.6$, there occurs a limit cycle.

$$
\begin{aligned}
r & =0.4, \\
k & =0.3, \\
d_{1} & =0.01, \\
d_{2} & =0.04, \\
m & =0.5, \\
c_{0} & =0.4, \\
d & =0.22, \\
a & =0.01 .
\end{aligned}
$$

Then, (64) has a positive equilibrium $E^{*}=(1.1122,0.579)$ and satisfies the conditions indicated in Theorem 3 ; then, $E^{*}=(1.1122,0.579)$ is asymptotically stable when $\tau=0$. Take $n=0$; we then have $\omega_{0}=0.2504$, $\tau_{0}=0.7003, \quad \mu_{2}=0.1746>0, \quad \beta_{2}=-0.0081<0, \quad$ and $T_{2}=0.2383>0$. Thus, $E^{*}$ is stable for $\tau<\tau_{0}$ as illustrated in Figure 6 . When $\tau$ passes through the critical value $\tau_{0}, E^{*}$ will lose its stability and a Hopf bifurcation will occurs; that is, a family of periodic solutions bifurcate from $E^{*}$. Since $\mu_{2}>0$, $\beta_{2}<0$, then the Hopf bifurcation is supercritical and its direction is $\tau>\tau_{0}$. These bifurcating periodic solutions from $E^{*}$ at $\tau_{0}$ are stable; see Figure 7 .

In Figure 8, we explore the effects of delay $\tau$ on the dynamics of system (64). Figure 8 shows that as $\tau$ increases, at first the positive equilibrium is stable; then when $\tau$ is greater than a certain critical value, periodic solution occurs; this is coincident with Theorem 3, which implies that delay destabilizes the equilibrium and induces oscillatory 


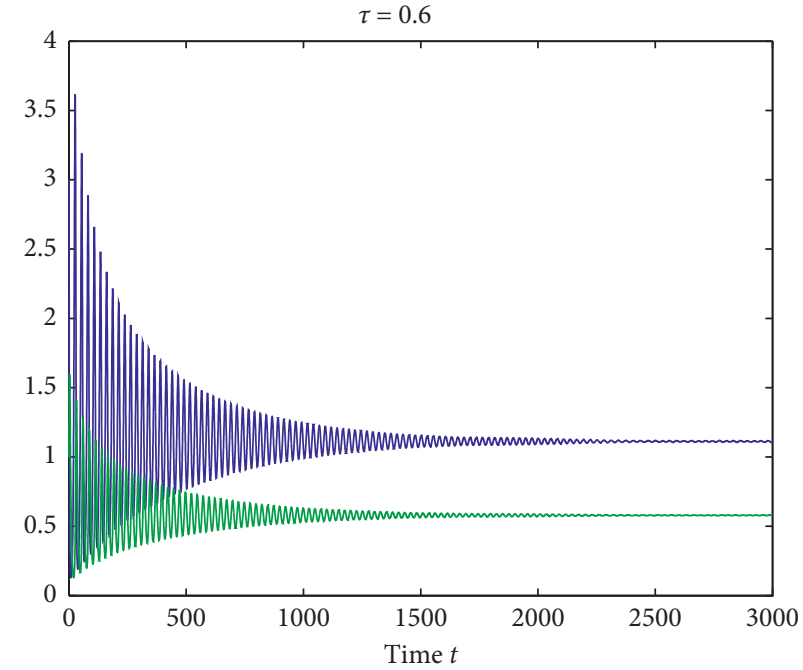

(a)

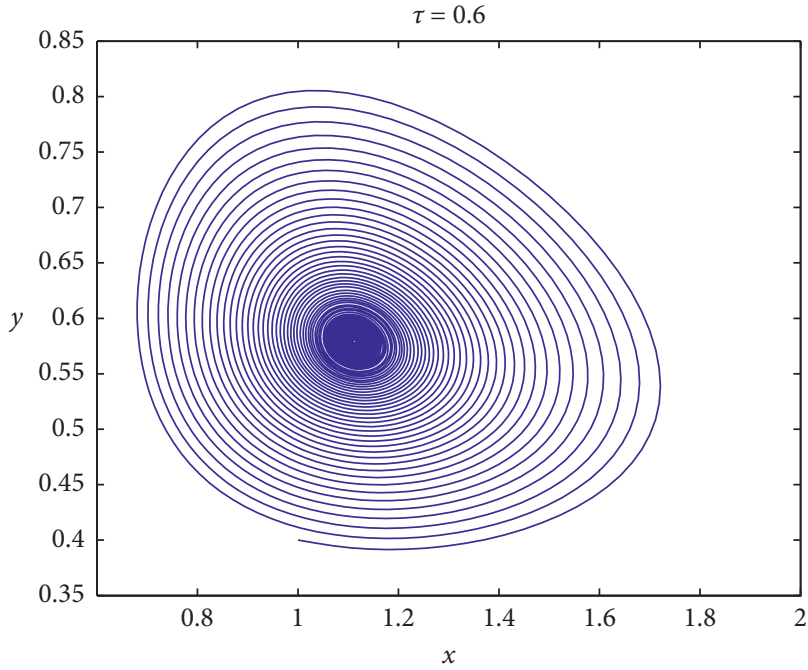

(b)

FIgURE 6: The figure shows that the dynamics of the system (64) of prey and predators. (a) represents the positive equilibrium $E^{*}=$ $(1.1122,0.579)$ that is locally asymptotically stable and (b) represents the phase-portrait of $E^{*}$ that is locally asymptotically stable when $\tau=0.6<\tau_{0}=0.7003$. The parameters are given in (65).

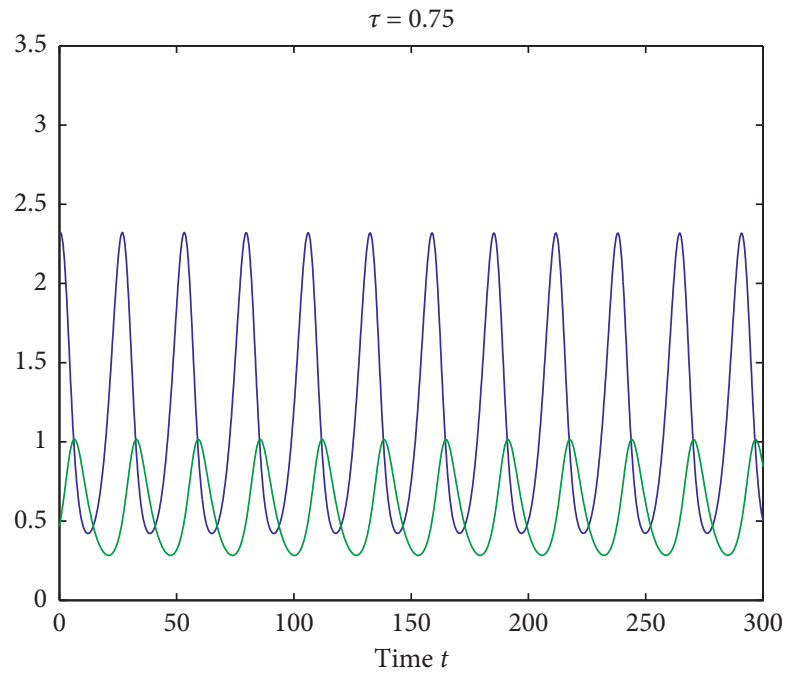

(a)

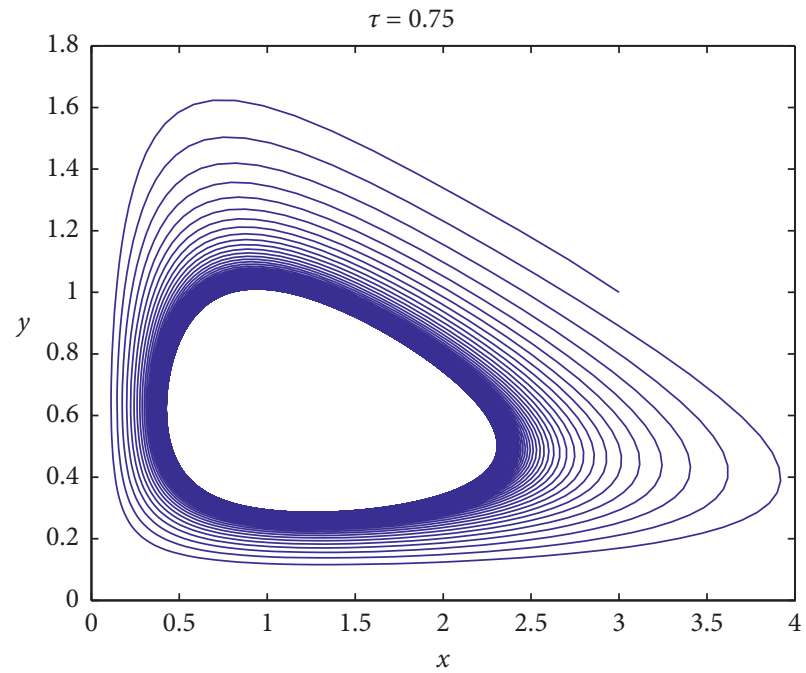

(b)

Figure 7: (a) represents the stable and periodic oscillations around the positive equilibrium $E^{*}=(1.1122,0.579)$ and the phase plane diagram (b) represents the stable limit cycle around $E^{*}$ when $\tau=0.75>\tau_{0}=0.7003$. The parameters are given in (65).

dynamics. By comparing Figure 3 and Figure 8, we see that the difference is big in the dynamics when the delay-dependent factor is incorporated or not.

In Figure 9, taking $k$ as a bifurcation parameter, we found that the fear effects can stabilize the system. In Figure 10, we draw the bifurcation diagram with respect to $m$; the shapes of the bifurcation diagrams for system (64) in Figure 10 and the original system (1) in Figure 5 are very similar.

\section{Discussion}

In this paper, taking into account the time delay corresponding to the gestation period and the effect that the fear of predators has on prey, we have proposed a predator-prey model with Holling-type II functional response. We have shown that the boundary equilibrium $E_{1}$ is globally asymptotically stable if (11) is reversed and pointed out that the instability of $E_{1}$ implies the permanence of the system 


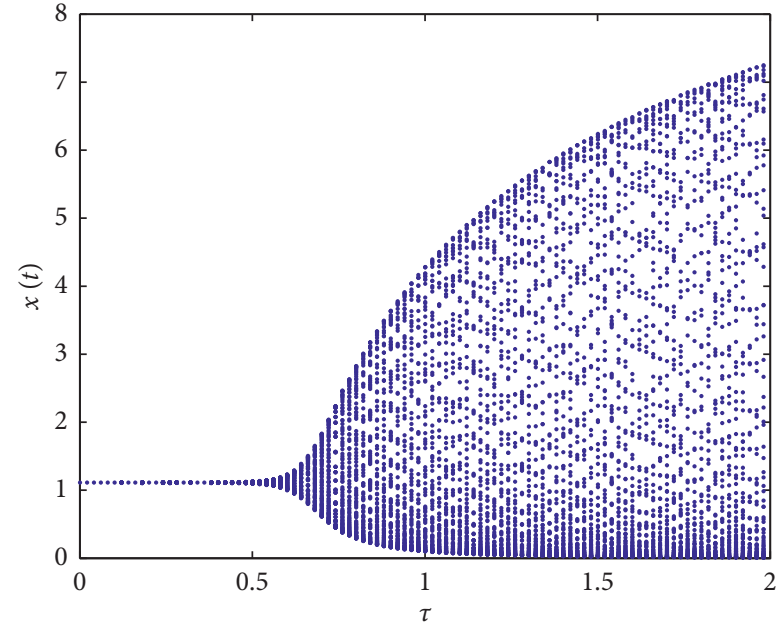

(a)

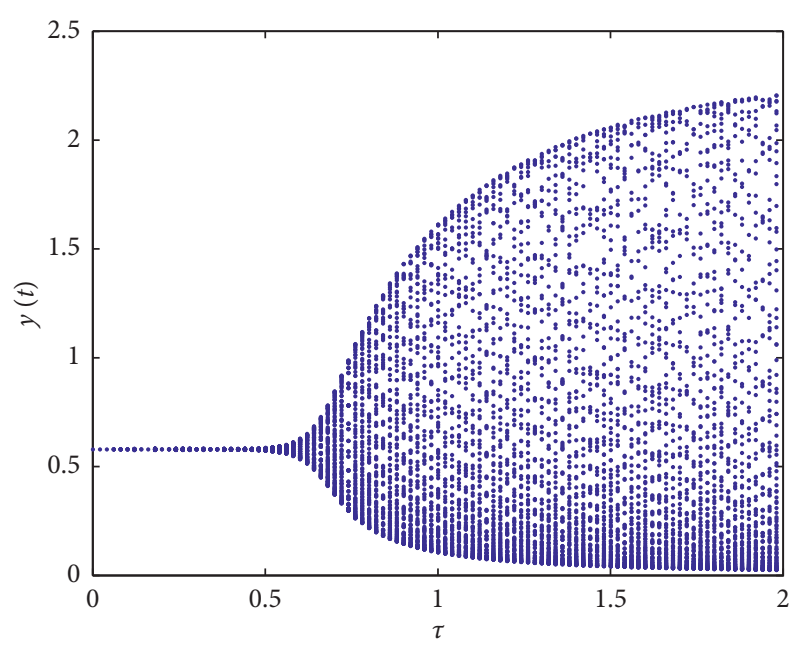

(b)

Figure 8: The figure shows the bifurcation diagrams of system (64) of prey and predator populations, which indicates the emergence of Hopf bifurcations as parameter $\tau$ increases, and other parameters are given in (65).

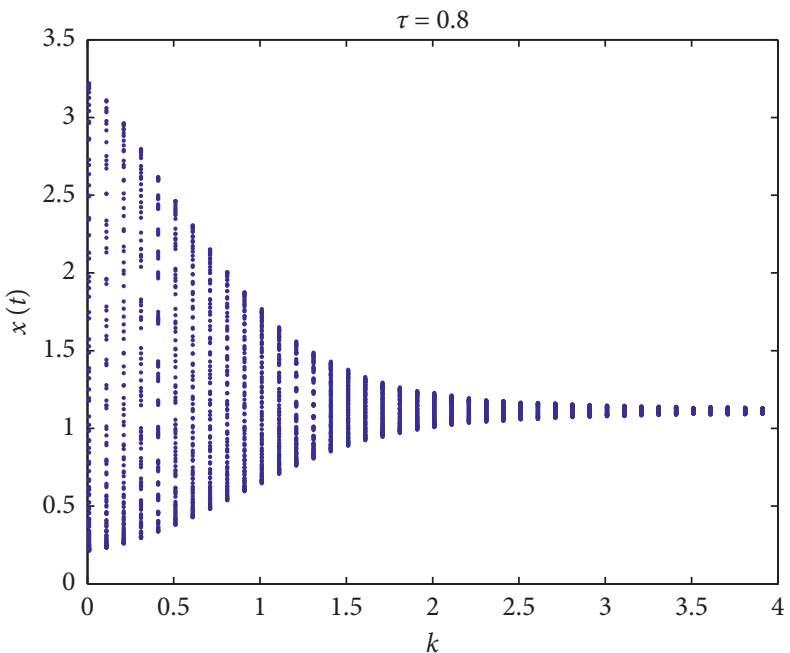

(a)

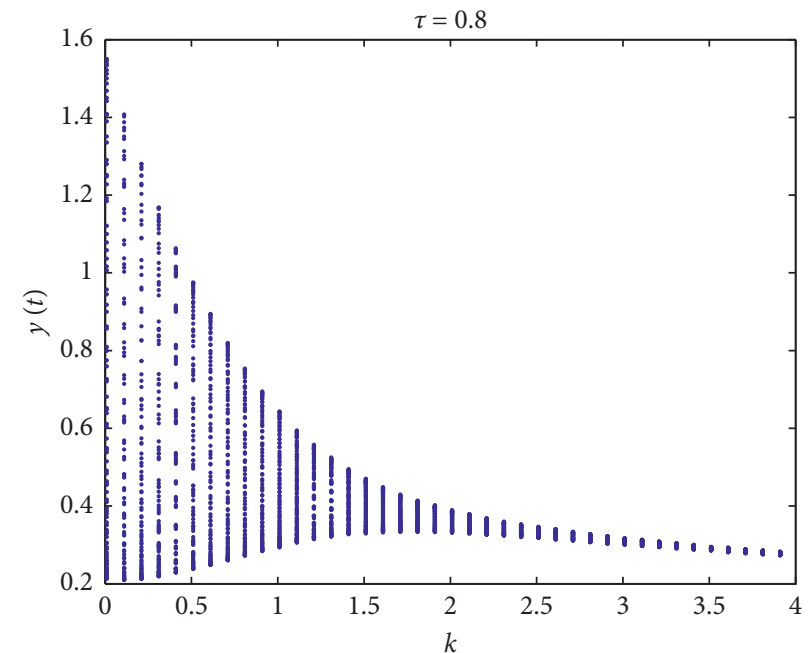

(b)

Figure 9: The figure shows the bifurcation diagrams of system (64) of prey and predator populations with respect to the parameter $k$, which shows that high levels of fear can stabilize the predator-prey system. However, relatively low levels of fear can induce oscillations via Hopf bifurcations, where $\tau=0.8$ and other parameters are given in (65).

and existence of the positive equilibrium $E^{*}$. Furthermore, for the system without delay-dependent factor, by choosing the delay $\tau$ as a bifurcation parameter, sufficient conditions for the existence of Hopf bifurcation have been presented. By using the theory of normal form and center manifold theorem, the explicit formulae determining the direction, stability, and other properties of bifurcating periodic solutions have been derived.

The novelty of this paper is that we have incorporated both the fear effect and a delay-dependent factor that ensures the predators that do not survive the delay interval do not contribute to the growth of the predator population. Our investigation shows that the dynamics of system (1) are affected significantly by not only the biomass transfer delay, but also the level of the fear effect. In [25], the delay-dependent factor was neglected.

Numerical simulations are performed to show the roles that time delay and fear effects can play in predator-prey interactions. For the original system (1), we have shown that both large time delay and high levels of fear (or equivalently strong antipredator responses) can stabilize the predatorprey system by excluding the existence of periodic solutions. And also, we observe that if the time delay as well as the fear level increases, the predator population goes to extinction. While for system (64) which neglects the delay-dependent factor, numerical simulations show that the delay has destabilizing effect while the large cost of fear has a stabilizing effect on the predator-prey interactions. Figure 8 


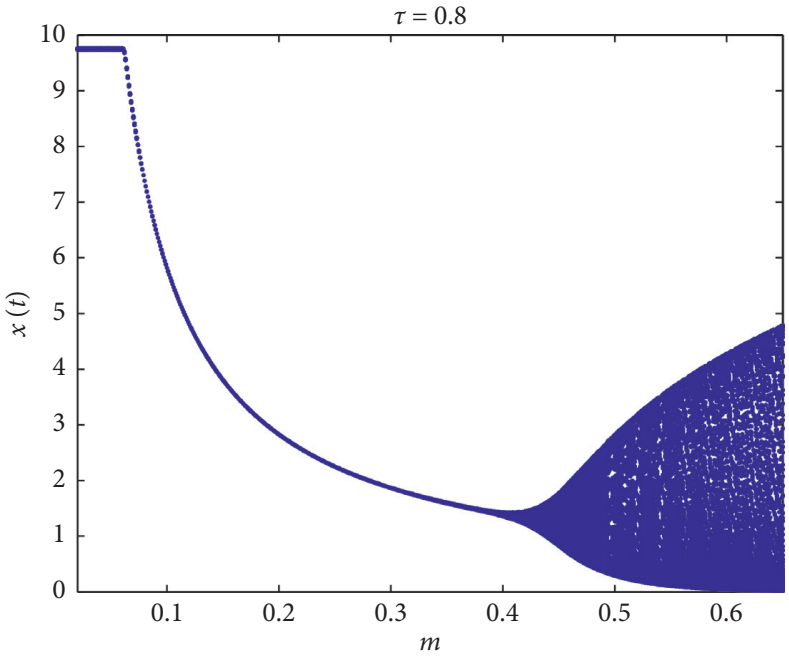

(a)

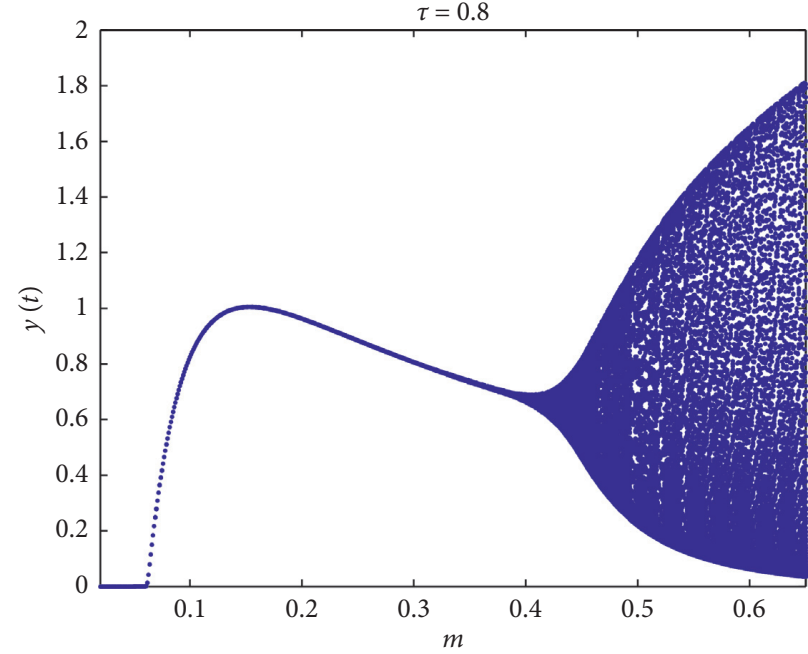

(b)

FIGURE 10: This figure shows the bifurcation diagrams of system (64) of prey and predators with respect to parameter $m$, which indicates the emergence of Hopf bifurcations as $k$ increases, where $\tau=0.8$ and other parameters are given in (65).

indicates that ignoring the delay-dependent factor results in model in (63) that does not predict the extinction of the predators, even if the delay is very large. Therefore, time delay and fear effect significantly influence the dynamics of the system and play important roles in ecosystems stability.

In the current paper, we assumed that the fear effects only reduce the birth rate of the prey, but it may also increase the death rate of adult prey due to long-term physiological impacts [11]. To make the model more realistic, some other important mechanisms can also be incorporated; for example, spatial effect is an important factor due to foraging behaviors of both prey and predator. The functional response plays a vital role in determining the different complex dynamical behaviors; different functional response functions can be chosen to reflect the group defense. It would be interesting and challenging to consider the aforementioned possible extensions; we leave these problems for further investigation.

\section{Data Availability}

All data generated or analyzed during this study are included in this article.

\section{Conflicts of Interest}

The authors declare that there are no conflicts of interest regarding the publication of this paper.

\section{Acknowledgments}

This work was supported by the National Natural Science Foundation of China (11801431), the International Cooperation on Science and Technology of the Key Research and Development Program of Shaanxi Province (2018KW-021), and the Natural Science Basic Research Plan in Shaanxi Province of China (2021JM-445).

\section{References}

[1] S. Creel and D. Christianson, "Relationships between direct predation and risk effects," Trends in Ecology \& Evolution, vol. 23, no. 4, pp. 194-201, 2008.

[2] F. Hua, K. E. Sieving, R. J. Fletcher, and C. A. Wright, "Increased perception of predation risk to adults and offspring alters avian reproductive strategy and performance," Behavioral Ecology, vol. 25, no. 3, pp. 509-519, 2014.

[3] W. Cresswell, "Predation in bird populations," Journal of Ornithology, vol. 152, no. 1, pp. 251-263, 2011.

[4] Z. Abramsky, M. L. Rosenzweig, and A. Subach, "The costs of apprehensive foraging," Ecology, vol. 83, no. 5, pp. 1330-1340, 2002.

[5] M. A. Elgar, "Predator vigilance and group size in mammals and birds: a critical review of the empirical evidence," Biological Reviews, vol. 64, no. 1, pp. 13-33, 1989.

[6] E. L. Preisser and D. I. Bolnick, "The many faces of fear: comparing the pathways and impacts of nonconsumptive predator effects on prey populations," PLoS One, vol. 3, no. 6, Article ID e2465, 2008.

[7] S. Creel, D. Christianson, S. Liley, and J. A. Winnie, "Predation risk affects reproductive physiology and demography of elk," Science, vol. 315, no. 5814, p. 960, 2007.

[8] D. S. Hik, "Does risk of predation influence population dynamics? Evidence from cyclic decline of snowshoe hares," Wildlife Research, vol. 22, no. 1, pp. 115-129, 1995.

[9] B. M. Pierce, R. T. Bowyer, and V. C. Bleich, "Habitat selection by mule deer: forage benefits or risk of predation?" Journal of Wildlife Management, vol. 68, no. 3, pp. 533-541, 2004.

[10] A. J. Wirsing and W. J. Ripple, "A comparison of shark and wolf research reveals similar behavioral responses by prey," Frontiers in Ecology and the Environment, vol. 9, no. 6, pp. 335-341, 2011.

[11] L. Y. Zanette, A. F. White, M. C. Allen, and M. Clinchy, "Perceived predation risk reduces the number of offspring songbirds produce per year," Science, vol. 334, no. 6061, pp. 1398-1401, 2011. 
[12] X. Wang, L. Zanette, and X. Zou, "Modelling the fear effect in predator-prey interactions," Journal of Mathematical Biology, vol. 73, no. 5, pp. 1179-1204, 2016.

[13] X. Wang and X. Zou, "Modeling the fear effect in predatorprey interactions with adaptive avoidance of predators," Bulletin of Mathematical Biology, vol. 79, no. 6, pp. 1325-1359, 2017.

[14] S. K. Sasmal and Y. Takeuchi, "Dynamics of a predator-prey system with fear and group defense," Journal of Mathematical Analysis and Applications, vol. 481, no. 1, Article ID 123471, 2020.

[15] P. Panday, N. Pal, S. Samanta, and J. Chattopadhyay, "Stability and bifurcation analysis of a three-species food chain model with fear," International Journal of Bifurcation and Chaos, vol. 28, no. 1, Article ID 1850009, 2018.

[16] K. Sarkar and S. Khajanchi, "Impact of fear effect on the growth of prey in a predator-prey interaction model," Ecological Complexity, vol. 42, Article ID 100826, , 2020.

[17] C. S. Holling, "The functional response of predators to prey density and its role in mimicry and population regulation," Memoirs of the Entomological Society of Canada, vol. 97, no. 45, pp. 1-60, 1965.

[18] H. L. Smith, Monotone Dynamical Systems, an Intoduction to the Theory of Competitive and Cooperative Systems, American Mathematical Society, Providence, RI, USA, 1995.

[19] Y. Xiao and L. Chen, "Modeling and analysis of a predatorprey model with disease in the prey," Mathematical Biosciences, vol. 171, no. 1, pp. 59-82, 2001.

[20] B. D. Hassard, N. D. Kazarinoff, and Y. H. Wan, Theory and Application of Hopf Bifurcation, Cambridge University Press, Cambridge UK, 1981.

[21] C. Sun, Y. Lin, and M. Han, "Stability and Hopf bifurcation for an epidemic disease model with delay," Chaos, Solitons \& Fractals, vol. 30, no. 1, pp. 204-216, 2006.

[22] J. Liu and T. Zhang, "Bifurcation analysis of an SIS epidemic model with nonlinear birth rate," Chaos, Solitons \& Fractals, vol. 40, no. 3, pp. 1091-1099, 2009.

[23] J. Liu and T. Zhang, "Hopf bifurcation and stability analysis for a stage-structured system," International Journal of Biomathematics, vol. 03, no. 01, pp. 21-41, 2010.

[24] J. K. Hale and P. Waltman, "Persistence in infinite-dimensional systems," SIAM Journal on Mathematical Analysis, vol. 20, no. 2, pp. 388-395, 1989.

[25] Y. Wang and X. Zou, "On a predator-prey system with digestion delay and anti-predation strategy," Journal of Nonlinear Science, vol. 30, no. 1, pp. 1579-1605, 2020. 